\title{
DISTAL FUNCTIONS ON GROUPS
}

\author{
BY \\ A. W. KNAPP( $\left.{ }^{1}\right)$
}

1. Introduction. Investigations of bounded complex-valued functions on groups have tied in closely in recent years with the study of the continuous functions on compact Hausdorff spaces on which groups act. Almost periodic functions, for example, can be treated as continuous functions on a compact group in which a homomorphic image of the given group is a dense subgroup, and the standard results about almost periodic functions then follow from the Peter-Weyl Theorem.

In 1963 Auslander and Hahn [1] reversed this situation by starting with functions on classes of compact spaces and using them to define classes of bounded functions on groups. For one example, they started with the class of so-called compact distal transformation groups, which had been introduced as early as 1902, and arrived at the class of distal functions, which are the subject of this paper.

Our intrinsic definition of distal functions is less easily motivated than the definition of distal transformation groups, but it is as follows: A bounded function $f$ on a group $G$ is right distal if whenever $\left\{g_{n}\right\},\left\{h_{i}\right\}$, and $\left\{k_{j}\right\}$ are nets in $G$ (functions from directed sets to $G$ ) with

$$
\lim _{n} \lim _{i} f\left(g g_{n} h_{i}\right)=\lim _{n} \lim _{j} f\left(g g_{n} k_{j}\right)
$$

pointwise for all $g$ in $G$, then already

$$
\lim _{i} f\left(g h_{i}\right)=\lim _{j} f\left(g k_{j}\right)
$$

In case $G$ is topological, we assume also that $f$ is left-uniformly continuous. Almost periodic functions are distal; functions on locally compact groups which vanish at infinity are not. On the integers $\exp \left(2 \pi i n^{2} \theta\right)$ is distal, and it is not almost periodic if $\theta$ is irrational. The set of all right distal functions will be shown to be a Banach algebra containing the constants and closed under conjugation and left and right translation.

The purpose of this paper is to give an analysis and synthesis of distal functions. The key to the results is the notion of relative almost periodicity, which is defined in $\$ 4$; we shall speak of functions which are almost periodic relative to a distal algebra-a left-invariant conjugate-closed Banach algebra of right distal functions containing the constants. The functions which are almost periodic over a distal

Received by the editors March 29, 1966.

(1) This research was supported in part by a fellowship from the National Science Foundation. The other part and the preparation of this paper were supported by Air Force Office of Scientific Research Grant AF-AFOSR 335-63. 
algebra themselves will form a distal algebra. The functions almost periodic over the algebra of constants will be exactly the almost periodic functions in the sense of von Neumann [12].

A distal algebra $A$ is defined to be almost periodic over a distal subalgebra $B$ if every function in $A$ is almost periodic over $B$. When this happens, there is a canonical $B$-valued invariant mean defined on $A$, i.e., a projection $E(\cdot \mid B)$ of $A$ onto $B$ which is $B$-linear, commutes with $G$, and sends functions $\geqq 0$ into functions $\geqq 0$.

The analysis and synthesis of distal functions proceeds in three steps, and the first two are given by Theorems 1.1 and 1.2.

THEOREM 1.1. If $G$ is locally compact $\sigma$-compact and if $A$ is a distal algebra, then there exist an ordinal $\nu$ and a system $A_{\xi}$ of distal subalgebras of $A$, indexed by all ordinals $0 \leqq \xi \leqq \nu$, such that

(a) $A_{0}$ is the algebra of constant functions, and $A_{v}=A$.

(b) If $\xi \leqq \xi^{\prime}$, then $A_{\xi} \subseteq A_{\xi^{\prime}}$.

(c) If $\xi<\nu$, then $A_{\xi+1}$ is a.p. over $A_{\xi}$.

(d) If $\xi$ is a limit ordinal $\leqq \nu$, then

$$
A_{\xi}=\text { unif. clos. }\left(\bigcup_{\eta<\xi} A_{\eta}\right) \text {. }
$$

THEOREM 1.2. If $G$ is any topological group and if $A$ is a distal algebra almost periodic over a distal subalgebra $B$, then there exists a set $\left\{P_{\lambda}\right\}$ of canonically determined bounded projection operators of $A$ into itself with the following properties:

(a) Image $\left(P_{\lambda}\right)$ is a finitely-generated B-module left-invariant under G. (More particularly, $P_{\lambda}$ is $B$-linear and commutes with the action of $G$.)

(b) (Orthogonality). If $\lambda \neq \sigma$ and if $f$ and $h$ are in $A$, then

$$
E\left(\left(P_{\lambda} f\right)\left(\overline{P_{\sigma} h}\right) \mid B\right)=0 .
$$

(c) (Completeness). For each $f$ in $A$, only countably many of the $P_{\lambda} f$ are different from 0 , and

$$
E\left(|f|^{2} \mid B\right)=\sum_{\lambda} E\left(\left|P_{\lambda} f\right|^{2} \mid B\right),
$$

the series on the right converging uniformly.

(d) (Approximation Theorem). There is a net of bounded B-linear G-commuting operators $T_{n}$ on $A$ with this property: If $P_{\lambda} f$ is nonzero for $\lambda=1,2,3, \ldots$, then $T_{n} f$ is in the weak direct sum

$$
\operatorname{image}\left(P_{1}\right) \oplus \operatorname{image}\left(P_{2}\right) \oplus \cdots
$$

and $\left\{T_{n} f\right\}$ converges uniformly to $f$.

(e) Every $G$-invariant $B$-submodule of image $\left(P_{\lambda}\right)$ is finitely generated, and image $\left(P_{\lambda}\right)$ splits into the finite direct sum of irreducible $G$-invariant $B$-modules.

(f) Call two finitely-generated $G$-invariant B-modules in $A$ equivalent if there is a bounded invertible B-linear map of one onto the other which commutes with the action of $G$. Then equivalent irreducible finitely-generated $G$-invariant B-modules are contained in the same image $\left(P_{\lambda}\right)$ and inequivalent ones are contained in different ones. 
In a special case Theorem 1.1 asserts that if we start with the constants, form all functions almost periodic over them, take all functions almost periodic over the resulting algebra, and keep going in this way (allowing uniform limits at limit ordinals), then eventually we get all right distal functions. The effect of Theorem 1.2 is to reduce the study of relative almost periodicity to the study of finitelygenerated $G$-invariant $B$-modules. (In fact, when $B$ is the algebra of constants, Theorem 1.2 simplifies to the usual analysis of almost periodic functions into finite-dimensional invariant vector spaces.) The two theorems taken together reduce the study of all distal functions to these $B$-modules.

The third step is to study these modules. To each such module we shall associate a vector bundle which makes it easier to understand. Introducing coordinates, we obtain a "unitary crossed representation" of $G$ from which the functions of the module can be recovered. Conversely if such a representation is given, then the functions it determines are all almost periodic over $B$ and generate such a $B$-module.

After the preliminaries, Theorem 1.1 is proved in $\$ 4$, Theorem 1.2 is proved in $\S \S 5-7$, and crossed representations are developed in $\$ 8$. Some examples are collected in $\$ 9$.

These results are an extension of those announced in [9] and of those in the author's doctoral thesis [10] written under the direction of Professor S. Bochner. I would like to express my appreciation particularly to Professors Bochner, $\mathrm{H}$. Furstenberg, and R. Gunning for their help with this work.

2. Notation and definitions. .Throughout this paper $G$ denotes a topological group with identity $e$. If $f$ is a complex-valued function on $G$, then $\|f\|$ denotes the supremum norm of $f$ and $f_{g}$ is the left translate of $f$ with $f_{g}(h)=f(g h)$. Such a function is left-uniformly continuous if $\lim _{g \rightarrow e}\left\|f_{g}-f\right\|=0$. The set of bounded leftuniformly continuous functions is denoted LUC; LUC is a conjugate-closed Banach algebra containing the constants and closed under left and right translation.

If $A$ is a Banach algebra in LUC containing the constants and closed under conjugation, then $A$ in a canonical way is isometric and algebraically isomorphic to the set of all continuous functions on the maximal ideal space $M(A)$ of $A$. If $g$ is in $G$, then the evaluation-at- $g$ homomorphism on $A$ has as its value on $f$ the constant $f(g)$. We shall use the letter $g$ in referring to the associated member of $M(A)$.

A flow $(X, G)$ will mean a jointly continuous group action of $G$ on the compact Hausdorff space $X$. (We shall suppose $G$ acts on the left.) If an algebra $A$ of the sort described above is also closed under left translation, then the left action of $G$ on itself extends in a natural way to a jointly continuous action of $G$ on $M(A)$, and $(M(A), G)$ is thereby a flow. Conversely if $(X, G)$ is a flow and if $p$ is a point of $X$, then for each continuous complex-valued function $f$ on $X$, the function $f_{1}$ defined on $G$ as $f_{1}(g)=f(g p)$ is in LUC, and the set of all such functions $f_{1}$ for fixed $p$ forms a conjugate-closed Banach algebra containing the constants, invariant under left translation, and having maximal ideal space the closure of the orbit of $p$ in $X$. We shall say $f_{1}$ arises from the orbit of $p$. (Normally, however, we shall use the 
same notation for a function on $X$ and the corresponding function on $G$.) The two constructions just described are inverse to one another if $p$ is chosen to be the evaluation-at-e functional in the maximal ideal space.

We recall from [11] the definition and properties of shift operators. Consider nets $\left\{g_{n}\right\}$ in $G$ such that $\lim _{n} f\left(g g_{n}\right)$ exists pointwise for every $g$ in $G$ and for every $f$ in LUC. Every convergent net, for instance, has this property. Call two such nets $\left\{g_{n}\right\}$ and $\left\{h_{m}\right\}$ equivalent if

$$
\lim _{n} f\left(g g_{n}\right)=\lim _{m} f\left(g h_{m}\right)
$$

pointwise for every $g$ in $G$ and $f$ in LUC. The operator that sends $f$ into its limiting value for that equivalence class is called the shift operator associated to the class. Shift operators are denoted $T_{\alpha}, T_{\beta}$, etc. Every right-translation operator is a shift operator.

Each shift operator is a homomorphism of norm one on LUC, each commutes with conjugation and left translation, and each has range in LUC. The composition of two shift operators is again a shift operator.

There is a one-one correspondence between the multiplicative linear functionals on LUC and the shift operators defined as follows: If a functional $\rho$ is given, put $T_{\alpha} f(g)=\rho\left(f_{g}\right)$. On the other hand, if a shift operator $T_{\alpha}$ is given, put $\rho(f)=T_{\alpha} f(e)$. The topology of $M$ (LUC) therefore induces a compact Hausdorff topology on the set of shift operators. In this topology a net of shift operators $\left\{T_{\alpha_{n}}\right\}$ converges to $T_{\alpha}$ if and only if $\left\{T_{\alpha_{n}} f\right\}$ converges to $T_{\alpha} f$ pointwise for every $f$ in LUC. Moreover, if $\lim T_{\alpha_{n}}=T_{\alpha}$, then $\lim T_{\alpha_{n}} T_{\beta}=T_{\alpha} T_{\beta}$ for fixed $T_{\beta}$ and hence the map $T_{\alpha} \rightarrow T_{\alpha} T_{\beta}$, for fixed $T_{\beta}$, is continuous. Thus the set of shift operators is a semigroup under composition and is a compact Hausdorff space, and its multiplication is continuous on one side. Note that the compactness implies the following: Any net in $G$ has a subnet which defines a shift operator.

If $(X, G)$ is a flow, the Ellis semigroup $\bar{G}$ of the flow is the closure in $X^{X}$ (endowed with the product topology) of the set of actions on $X$ by the members of $G$. It is clear that $\bar{G}$ is compact Hausdorff, and it is shown in [5] that $\bar{G}$ is a semigroup in which the map $t \rightarrow t s$ for fixed $s$ is continuous.

In [11] a connection was shown between the set of shift operators and Ellis semigroups. Let $A$ be a conjugate-closed Banach algebra in LUC containing the constants and invariant under left translation. Relative to $A$ we define an equivalence relation $\sim$ on the set of shift operators. Call $T_{\alpha} \sim T_{\beta}$ if $T_{\alpha} T_{\gamma} f=T_{\beta} T_{\gamma} f$ for all $f \in A$ and for all $T_{\gamma}$. The quotient inherits the semigroup structure of the set of shift operators, and it is compact Hausdorff in the quotient topology. If $t$ is in the Ellis semigroup of $M(A)$, put $T f(g)=f_{g}(t e)$ for $g \in G$ and $f \in A$; the result $t \rightarrow T$ can be shown to be a function (referred to below as the natural map) into the set of equivalence classes of shift operators. Theorem 4-2 of [11] becomes

Lemma 2.1. Let $A$ be a conjugate-closed Banach algebra in LUC containing the constants and invariant under left translation. Then the natural map of the Ellis 
semigroup of $M(A)$ is a topological isomorphism onto the quotient of the set of shift operators by the equivalence relation induced by $A$.

A flow $(Y, G)$ is a subflow of $(X, G)$ with respect to $\pi$ if $\pi$ is a continuous map of $X$ onto $Y$ which commutes with the action of $G$. If $B$ and $A$ are Banach algebras in LUC containing the constants and closed under conjugation and left translation and if $B \subseteq A$, then the inclusion of algebras induces a continuous projection of $M(A)$ onto $M(B)$ which carries the $e$ of $M(A)$ onto the $e$ of $M(B)$. Moreover $\pi$ commutes with the action of $G$ and thus exhibits $M(B)$ as a subflow of $M(A)$.

The following lemma which connects subflows and Ellis semigroups, was pointed out as part of Lemma 2 by Ellis and Gottschalk in [6]. It will be used frequently without specific reference.

Lemma 2.2. If $(X, G)$ is a flow with Ellis semigroup $\bar{G}$ and if $(Y, G)=\pi(X, G)$ is a subflow, then, for any $x_{1}$ and $x_{2}$ in $X, \pi\left(x_{1}\right)=\pi\left(x_{2}\right)$ implies $\pi\left(t x_{1}\right)=\pi\left(t x_{2}\right)$ for all $t$ in $\bar{G}$.

3. Distal functions. A function $f$ in LUC is right distal if whenever

$$
\lim _{n} \lim _{i} f\left(g g_{n} h_{i}\right)=\lim _{n} \lim _{j} f\left(g g_{n} k_{j}\right)
$$

for some three nets in the group and for all $g$, then also

$$
\lim _{i} f\left(g h_{i}\right)=\lim _{j} f\left(g k_{j}\right) .
$$

In view of the compactness of the set of shift operators, $f$ is right dis $\uparrow 1$ if and only if an equality $T_{\alpha} T_{\beta} f=T_{\alpha} T_{\gamma} f$ always implies $T_{\beta} f=T_{\gamma} f$.

Right almost periodic functions (right and left are actually equivalent) are right distal because the convergence is uniform in (3.1). Hence

$$
\lim _{n}\left\|\lim _{i} f\left(g g_{n} h_{i}\right)-\lim _{j} f\left(g g_{n} k_{j}\right)\right\|=0
$$

or, for any $\varepsilon>0$,

$$
\left\|\lim _{i} f\left(g g_{n} h_{i}\right)-\lim _{j} f\left(g g_{n} k_{j}\right)\right\|<\varepsilon
$$

eventually. Replacing $g$ by $g g_{n}^{-1}$, we obtain

$$
\left\|\lim _{i} f\left(g h_{i}\right)-\lim _{j} f\left(g k_{j}\right)\right\|<\varepsilon .
$$

Since $\varepsilon$ is arbitrary, $f$ is right distal.

On the other hand it is shown in $\S 9$ that, for any real number $\theta$, the function $\exp \left(i n^{2} \theta\right)$ on the integers is distal. But if $\theta$ is an irrational multiple of $\pi$, this function is easily seen to have all Bohr-Fourier coefficients equal to 0 , and hence it is not almost periodic.

Left distal functions will play no role in this paper, and we shall therefore use distal to mean right distal. (We warn the reader that we do not know whether right 
distal and left distal are the same.) A distal algebra is a Banach algebra of distal functions containing the constants and closed under conjugation and left translation.

Our first step is to show that the distal functions are exactly the functions arising from distal flows. A flow $(X, G)$ is distal if whenever $x, y$, and $z$ are in $X$ and $\left\{g_{n}\right\}$ is a net in $G$ such that

$$
g_{n} x \rightarrow z \text { and } g_{n} y \rightarrow z,
$$

then $x=y$. The lemma and two corollaries below were proved by Ellis [4] and re-proved by Furstenberg [8]. These results are central to this paper.

Lemma 3.1. A flow $(X, G)$ is distal if and only if its Ellis semigroup is a group.

Corollary 3.2. A subflow of a distal flow is distal.

COROLlaRY 3.3. If $(X, G)$ is a distal flow with a dense G-orbit, then every G-orbit is dense and the Ellis group acts transitively on $X$.

If $f$ is a member of LUC, let $A_{f}$ be the least Banach algebra containing $f$, its left translates, their complex conjugates, and the constant functions.

Proposition 3.4. If $f$ is in LUC, then $f$ is distal if and only if $M\left(A_{f}\right)$ is a distal flow.

Proof. If $f$ is distal, we show $M\left(A_{f}\right)$ is a distal flow. Let $z=\lim g_{n} x=\lim g_{n} y$, $x=\lim h_{i}$, and $y=\lim k_{j}$. The last two equations are possible since the evaluations are dense in $M\left(A_{f}\right)$. Then for every $g$ in $G$

$$
f_{g}(z)=\lim _{n} \lim _{i} f_{g}\left(g_{n} h_{i}\right)=\lim _{n} \lim _{j} f_{g}\left(g_{n} k_{j}\right)
$$

and hence (3.1) holds. Since $f$ is distal, (3.2) gives

$$
f_{g}(x)=f(g x)=\lim _{i} f_{g}\left(h_{k}\right)=\lim _{j} f_{g}\left(k_{j}\right)=f(g y)=f_{g}(y) .
$$

Since the left translates of $f$ separate points on $M\left(A_{f}\right)$, we obtain $x=y$. The converse follows from the implication (b) $\Rightarrow$ (a) in

Proposition 3.5. If $A$ is a Banach algebra in LUC containing the constants and closed under conjugation and left translation, then the following conditions are equivalent:

(a) Every function in $A$ is distal.

(b) $M(A)$ is a distal flow.

(c) $M\left(A_{f}\right)$ is a distal flow for every $f$ in $A$.

Proof. (a) $\Rightarrow$ (b). Repeat the proof of Proposition 3.4 and use the fact that the members of $A$ separate points on $M(A)$.

(b) $\Rightarrow$ (c). Corollary 3.2 .

(c) $\Rightarrow$ (a). If $M\left(A_{f}\right)$ is distal, then the shift-operator classes for $A_{f}$ form a group 
by Lemmas 2.1 and 3.1. Let $T_{\alpha} T_{\beta} f=T_{\alpha} T_{\gamma} f$ and let $\left[T_{\varepsilon}\right]\left[T_{\alpha}\right]$ be the identity. This stipulation means that

$$
T_{\beta} f=T_{\varepsilon} T_{\alpha} T_{\beta} f=T_{\varepsilon} T_{\alpha} T_{\gamma} f=T_{\gamma} f .
$$

Hence $f$ is distal, and the proof is complete.

Conditions (b) and (c) of Proposition 3.5 were shown to be equivalent by Auslander and Hahn in [1]. They took these conditions as a definition of a class of functions, and they proved this class was a lattice algebra. We see that they were dealing with the set of all distal functions. We show now that this set is actually a Banach algebra.

We require some results proved by Ellis. In Lemma 3.6 the three statements are from Lemma 1 in [4] and Lemmas 1 and 2 in [5]. A left ideal in a semigroup $S$ is a nonempty subset $I$ such that $S I \subseteq I$.

LEMMA 3.6. Let $W$ be a compact Hausdorff space with a semigroup structure such that the maps $t \rightarrow t$ are continuous for each $s$. Then

(a) Each nonempty closed subset I of $W$ with $I^{2} \subseteq I$ contains an idempotent.

(b) The minimal closed left ideals are the same as the minimal left ideals.

(c) If I is a minimal left ideal of $W$ and if $p \in I$, then there exist $s$ and $u$ in I such that $u$ is an idempotent, $u p=p$, and $p s=s p=u$.

A shift operator $T_{\alpha}$ is minimal if $T_{\alpha}$ is in some minimal left ideal of the set of all shift operators. (This definition is not identical with the one in the author's announcement [9], but it is not hard to show that the definitions are equivalent.) The following was announced as Theorem 1 in [9].

THEOREM 3.7. If $f$ is in LUC, the following are equivalent:

(a) $f$ is distal.

(b) $T_{u} T_{\alpha} f=T_{\alpha}$ ffor every $T_{\alpha}$ and for every idempotent $T_{u}$.

(c) $T_{u} T_{\alpha} f=T_{\alpha}$ ffor every $T_{\alpha}$ and for every minimal idempotent $T_{u}$.

Proof. (a) $\Rightarrow$ (b). We have $T_{u} T_{u} T_{\alpha} f=T_{u} T_{\alpha} f$. If $f$ is distal, $T_{u} T_{\alpha} f=T_{\alpha} f$.

(b) $\Rightarrow$ (c). A fortiori.

(c) $\Rightarrow$ (a). Order the closed nonempty left ideals of the set of all shift operators by inclusion downward, and by Zorn's Lemma let $I$ be a minimal one. Then $I$ is a minimal left ideal by part (b) of Lemma 3.6 and $I$ contains an idempotent $T_{v}$ by part (a) of that lemma. Therefore a minimal idempotent $T_{v}$ exists. Now let $T_{\alpha} T_{\beta} f$ $=T_{\alpha} T_{\gamma} f$. By hypothesis

and hence

$$
T_{v} T_{\beta} f=T_{\beta} f \quad \text { and } \quad T_{v} T_{\gamma} f=T_{\gamma} f
$$

$$
T_{\alpha} T_{v} T_{\beta}=T_{\alpha} T_{v} T_{\gamma} f
$$

But $T_{\alpha} T_{v}$ is in $I$ since $I$ is a left ideal. Apply part (c) of Lemma 3.6 to find a member $T_{\varepsilon}$ of $I$ and an idempotent $T_{u}$ of $I$ with

$$
T_{u}\left(T_{\alpha} T_{v}\right)=T_{\alpha} T_{v} \quad \text { and } \quad T_{\varepsilon}\left(T_{\alpha} T_{v}\right)=T_{u}=\left(T_{\alpha} T_{v}\right) T_{\varepsilon}
$$


Then

$$
T_{u} T_{\beta} f=T_{\varepsilon} T_{\alpha} T_{v} T_{\beta} f=T_{\varepsilon} T_{\alpha} T_{v} T_{\gamma} f=T_{u} T_{y} f,
$$

and it follows that $T_{\beta} f=T_{\gamma} f$ by two applications of the hypothesis. Hence $f$ is distal.

COROLlaRY 3.8. The set of all distal functions on $G$ is a Banach algebra containing the constants and closed under conjugation and left translation. Any shift of a distal function is distal.

Proof. Immediate from condition (b) of Theorem 3.7.

4. Relative almost periodicity. The key to the results of this paper is the definition of relative almost periodicity, which is given in this section together with a proof of Theorem 1.1.

Let $B$ be a distal algebra. We say that a function $f$ in LUC is almost periodic relative to $B$ (a.p. over $B$ ) if $f$ is distal and if whenever the conditions

$$
T_{\alpha_{n}} \rightarrow T_{\alpha} \text { and } T_{\delta_{n}} \rightarrow T_{\delta}
$$

and

$$
T_{\delta_{n}} h=h=T_{\delta} h
$$

for all $h$ in $B$ and for all $n$ are satisfied, then

$$
T_{\alpha_{n}} T_{\delta_{n}} \rightarrow T_{\alpha} T_{\delta} f \text { pointwise. }
$$

A distal algebra $A$ is a.p. over $B$ if $A \supseteq B$ and if every function in $A$ is a.p. over $B$. We denote by $A P(B)$ the set of all functions a.p. over $B$.

We shall see in Corollary 4.6 that $A P$ (constants) is the set of all almost periodic functions on $G$. In fact, when $B$ is the algebra of constants, (4.2) is vacuous, and (4.3) is not very different from the definition of almost periodicity given in Bochner [2].

The first step in proving Theorem 1.1 is the characterization in Proposition 4.4 of almost periodicity of $A$ over $B$ in terms of the Ellis group of $M(A)$.

Proposition 4.1. If $B$ is a distal algebra, then $A P(B)$ is a distal algebra with $A P(B) \supseteq B$.

Proof. $A P(B)$ is a distal algebra because the distal functions form a distal algebra and because (4.3) is preserved under all the operations defining a distal algebra. If $h$ is in $B$, then $h$ is distal and (4.3) is satisfied because

$$
T_{\alpha_{n}} T_{\delta_{n}} h=T_{\alpha_{n}} h \rightarrow T_{\alpha} h=T_{\alpha} T_{\delta} h .
$$

Hence $A P(B) \supseteq B$.

Lemma 4.2. Let $B$ be a distal algebra, let $T_{\beta_{n}}$ and $T_{\beta}$ be shift operators with 
$T_{\beta_{n}} \rightarrow T_{\beta}$, and let $T_{\varepsilon_{n}}$ and $T_{\varepsilon}$ be shift operators with $T_{\varepsilon_{n}} \rightarrow T_{\varepsilon}$ and $T_{\varepsilon_{n}} T_{\gamma} h=T_{\gamma} h$ $=T_{\varepsilon} T_{\gamma} h$ for all $h$ in $B$ and some fixed $T_{\gamma}$. If $f$ is a.p. over $B$, then

$$
T_{\beta_{n}} T_{\varepsilon_{n}} T_{\gamma} f \rightarrow T_{\beta} T_{\varepsilon} T_{\gamma} f
$$

Proof. Since the distal functions form a distal algebra (Corollary 3.8), their maximal ideal space is a distal flow (Proposition 3.5) whose Ellis semigroup is a group (Lemma 3.1). Thus the isomorphism given in Lemma 2.1 shows that there is a shift operator $T_{\gamma}^{-1}$ which is inverse to $T_{\gamma}$ on the set of all distal functions. Put $T_{\delta_{n}}=T_{\gamma}^{-1} T_{\varepsilon_{n}} T_{\gamma}$ and $T_{\delta}=T_{\gamma}^{-1} T_{\varepsilon} T_{\gamma}$. Then $T_{\delta_{n}}$ and $T_{\delta}$ satisfy (4.2). Put $T_{\alpha_{n}}=T_{\beta_{n}} T_{\gamma}$ and $T_{\alpha}=T_{\beta} T_{\gamma}$. Then $T_{\alpha_{n}} \rightarrow T_{\alpha}$. Passing to a subnet if necessary, we may suppose that $T_{\delta_{n}}$ converges. If we can show that $T_{\delta_{n}} f \rightarrow T_{\delta} f$, then (4.3) gives

$$
T_{\beta_{n}} T_{\varepsilon_{n}} T_{\gamma} f=T_{\alpha_{n}} T_{\delta_{n}} f \rightarrow T_{\alpha} T_{\delta} f=T_{\beta} T_{\varepsilon} T_{\gamma} f
$$

as required.

Thus suppose $T_{\delta_{n}} \rightarrow T_{\tau}$. By relative almost periodicity of $f$, we have

Therefore

$$
T_{\gamma} T_{\tau} f=\lim T_{\gamma} T_{\delta_{n}} f=\lim T_{\varepsilon_{n}} T_{y} f=T_{\varepsilon} T_{\gamma} f
$$

$$
\lim T_{\delta_{n}} f=T_{\tau} f=T_{\gamma}^{-1} T_{\varepsilon} T_{\gamma} f=T_{\delta} f,
$$

and the proof is complete.

From here through Corollary 4.5 let $A$ and $B$ be fixed distal algebras with $A \supseteq B$, let $\bar{G}$ be the Ellis group of $M(A)$, and let $\pi$ be the projection of $M(A)$ onto $M(B)$. We denote by $X_{y}$ the fiber $\pi^{-1}(y)$ and by $\bar{I}_{y}$ the set of all $t$ in $\bar{G}$ such that $\pi(t x)=\pi(x)$ for all $x \in X_{y}$, i.e., the set of all $t$ which leave the fiber $X_{y}$ fixed as a set. If the actions of the elements of $\bar{I}_{y}$ are restricted to $X_{y}$ and elements are identified which have the same action on $X_{y}$, the resulting quotient space will be called $I_{y} ; I_{y}$ is a set of point transformations of $X_{y}$ and is given the quotient topology.

LEMMA 4.3. If $t$ in $\bar{G}$ maps some point $x_{1}$ of $X_{y}$ into a point of the same fiber, then $t$ is in $\bar{I}_{y}$.

Proof. If $x$ is such that $\pi(x)=\pi\left(x_{1}\right)$, then $\pi(t x)=\pi\left(t x_{1}\right)$ by Lemma 2.2 .

Proposition 4.4. Let $A$ and $B$ be distal algebras with $A \supseteq B$. Then $A$ is a.p. over $B$ if and only if the following condition is satisfied: For each $y$ in $M(B)$, whenever

$$
t_{n} \text { and } t \text { are in } \bar{G} \text { with } t_{n} \rightarrow t \text { and }
$$

$$
s_{n} \text { and } s \text { are in } \bar{I}_{y} \text { with } s_{n} \rightarrow s \text {, }
$$

then

$$
t_{n} s_{n}(x) \rightarrow t s(x) \text { for all } x \text { in } \pi^{-1}(y) .
$$

Proof. We first remark that if the quotient map on the set of shift operators (by the relation induced by $A$ ) is followed by the isomorphism of Lemma 2.1 , then the 
result is a continuous map of the set of shift operators onto $\bar{G}$ such that the image $u$ of $T_{\gamma}$ satisfies $f(u e)=T_{y} f(e)$. Moreover, if two shift operators are given, then

$$
f\left(u_{1} u_{2} e\right)=T_{\gamma_{1}} T_{\gamma_{2}} f(e) .
$$

Let the condition of the proposition be satisfied. Under the above map let $T_{\delta_{n}}$ go into $s_{n}, T_{\delta}$ go into $s, T_{\alpha_{n}}$ go into $t_{n}$, and $T_{\alpha}$ go into $t$. Then $s_{n} \rightarrow s$ and $t_{n} \rightarrow t$. If $h$ is in $B$ and $x$ is in $\bar{I}_{e}$, then $h\left(s_{n} e\right)=T_{o_{n}} h(e)=h(e)$. Since such $h$ 's separate points of $M(B), \pi\left(s_{n} e\right)=\pi(e)$. By Lemma $4.3 s_{n}$ is in $\bar{I}_{e}$. Similarly $s$ is in $\bar{I}_{e}$ and therefore $t_{n} s_{n}(e) \rightarrow t s(e)$ and $f\left(t_{n} s_{n} e\right) \rightarrow f(t s e)$. This result applied to $f_{g}$ gives

$$
f_{g}\left(t_{n} s_{n} e\right) \rightarrow f_{g}(t s e) .
$$

The remark at the end of the above paragraph then shows that (4.3) holds.

Conversely, let $A$ be a.p. over $B$, and let $y, s_{n}, s, t_{n}$, and $t$ be given. Lift $s_{n}$ to some $T_{\varepsilon_{n}}$ and $t_{n}$ to some $T_{\beta_{n}}$ under the composition map in the first paragraph of the proof. By compactness it is enough to prove that $t_{n} s_{n}(x) \rightarrow t s(x)$ for subnets along which $T_{\varepsilon_{n}}$ and $T_{\beta_{n}}$ are both convergent. We may therefore assume that $T_{\varepsilon_{n}} \rightarrow T_{\varepsilon}$ and $T_{\beta_{n}} \rightarrow T_{\beta}$ to begin with. Then $T_{\varepsilon}$ projects to $s$ and $T_{\beta}$ projects to $t$ by continuity. Let $u \in \bar{G}$ send $e$ into $x$, and choose $T_{\gamma}$ projecting to $u$. For $f$ in $A$ we have

$$
T_{\varepsilon_{n}} T_{\gamma} f(e)=f\left(s_{n} u e\right)=f\left(s_{n} x\right) .
$$

Thus for $h \in B$,

$$
\begin{aligned}
T_{\varepsilon_{n}} T_{\gamma} h(g) & =T_{\varepsilon_{n}} T_{\gamma} h_{g}(e)=h_{g}\left(s_{n} x\right)=h_{g}\left(\pi\left(s_{n} x\right)\right) \\
& =h_{g}(\pi(x))=h_{g}(x)=T_{\gamma} h_{g}(e)=T_{\gamma} h(g),
\end{aligned}
$$

or $T_{\varepsilon_{n}} T_{\gamma} h=T_{\gamma} h$. Similarly $T_{\varepsilon} T_{\gamma} h=T_{\gamma} h$. By Lemma 4.2

$$
f\left(t_{n} s_{n} x\right)=T_{\beta_{n}} T_{\varepsilon_{n}} T_{\gamma} f(e) \rightarrow T_{\beta} T_{\varepsilon} T_{y} f(e)=f(t s x)
$$

for all $f$ in $A$. Then $t_{n} s_{n} x \rightarrow t s x$ since the $f$ 's separate points on $M(A)$.

Corollary 4.5. If $A$ and $B$ are distal algebras with $A$ almost periodic over $B$, then $I_{y}$ is a compact topological group and the action of $I_{y}$ on $X_{y}$ is jointly continuous and transitive.

Proof. If $t_{n}$ is in $\bar{I}_{y}$ and $t_{n} \rightarrow t$, then, for $x$ in $X_{y}, \pi(x)=\pi\left(t_{n} x\right) \rightarrow \pi(t x)$ and hence $t$ is in $\bar{I}_{y}$. Thus $\bar{I}_{y}$ is compact and so is $I_{y}$. To show that $I_{y}$ is Hausdorff, we must show that the equivalence relation on $\bar{I}_{y}$ is closed. Let $s_{n} \sim t_{n}, s_{n} \rightarrow s$, and $t_{n} \rightarrow t$. Then, for $x$ in $X_{y}, s_{n} x=t_{n} x$. Passing to the limit, we get $s x=t x$ and hence $s \sim t$. Therefore $I_{y}$ is Hausdorff.

Next we show that $I_{y}$ is a group. It is clear that $\bar{I}_{y}$ is a semigroup. If $t$ is in $\bar{I}_{y}$, fix an $x_{0}$ in $X_{y}$. Then $t x_{0}$ is in $X_{y}$ and so is $t^{-1}\left(t x_{0}\right)=x_{0}$. By Lemma 4.3, $t^{-1}$ is in $\bar{I}_{y}$, and $\bar{I}_{y}$ is a group. Let $s, t$, and $u$ be in $\bar{I}_{y}$ with $s \sim t$. Then $s x=t x$ for all $x$ in $X_{y}$, in particular for $u x$. Thus $s u \sim t u$. Since $s x$ and $t x$ are in $X_{y}$, we also have $u s x=u t x$. Thus us ut. Consequently $I_{y}$ is a group. 
Convergence in $I_{y}$ is pointwise convergence on $X_{y}$ of the transformations of $X_{y}$. Proposition 4.4 therefore shows that multiplication is jointly continuous. Since the map $(x, z) \rightarrow(x, x z)$ of $I_{y} \times I_{y}$ into itself is continuous, one-one, and onto, its inverse $(x, z) \rightarrow\left(x, x^{-1} z\right)$ is continuous. The map $x \rightarrow x^{-1}$ is then the composition of the continuous functions

$$
x \rightarrow(x, z) \rightarrow\left(x, x^{-1} z\right) \rightarrow x^{-1} z \rightarrow x^{-1},
$$

and $I_{y}$ is a compact group.

The action of $I_{y}$ is transitive on $X_{y}$ because if $x_{0}$ and $x$ are in $X_{y}$, let $t$ be an element of $\bar{G}$ with $t x_{0}=x$ (existence by Corollary 3.3). By Lemma 4.3, $t$ is in $\bar{I}_{y}$, and hence $[t]$ is an element of $I_{y}$ mapping $x_{0}$ into $x$.

Finally the action of $I_{y}$ on $X_{y}$ is jointly continuous because a compact group which acts on a compact space under the topology of pointwise convergence always acts in a jointly continuous manner.

COROLlary 4.6. The functions almost periodic over the algebra of constant functions are exactly the ordinary almost periodic functions.

Proof. If $f$ is almost periodic, then $f$ is distal and (4.2) holds for all shift operators. The shift operators may be identified in a many-one way with translations on the Bohr compactification, and the fact that (4.1) implies (4.3) is a reflection of the joint continuity of the multiplication on the compactification.

Conversely, Proposition 4.1 shows that we may apply Corollary 4.5 with $A=A P$ (constants) and $B=$ constants. That corollary shows that $\bar{G}$ is a compact topological group and $M(A)$ is a homogeneous space. The proof that the functions in $A$ are almost periodic is then routine.

We come now to the proof of Theorem 1.1. The system $\left\{A_{\xi}\right\}$ of the theorem will be called a transfinite sequence associated to $A$, and the ordinal $v$ is the length of the sequence.

Lemma 4.7. Let $A$ be a distal algebra, and suppose that, for each $f$ in $A, A_{f}$ has a transfinite sequence of length $\nu_{f}$. Then $A$ has a transfinite sequence of length $\sup _{f \in A} \nu_{f}$.

Proof. Let $A_{f}(\xi)$ be the $\xi$ th step algebra in the sequence for $A_{f}$. (For ordinals from $\nu_{f}+1$ to $\sup _{f \in A} \nu_{f}$, we let $A_{f}(\xi)=A_{f}$.) Let $A(\xi)$ be the least Banach algebra containing all $A_{f}(\xi)$ as $f$ ranges over $A$. We shall prove by transfinite induction on $\xi$ that $A(\xi)$ is a transfinite sequence for $A$. We note that conditions (a) and (b) are clearly satisfied. Thus let $\xi$ be given, and suppose that for all $\eta<\xi$ conditions (c) and (d) are satisfied.

Case $1 . \xi$ is a limit ordinal.

We are to prove that

$$
A(\xi)=\text { unif. clos. }\left[\bigcup_{\eta<\xi} A(\eta)\right]
$$

Containment $\supseteq$ is evident because

$$
A(\xi) \supseteq A_{f}(\xi) \supseteq A_{f}(\eta) \text { for all } \eta<\xi \text { and all } f
$$


and hence

and

$$
A(\xi) \supseteq \bigvee_{f} A_{f}(\eta)=A(\eta) \quad \text { for all } \eta<\xi
$$

$$
A(\xi) \supseteq \operatorname{cl}[\bigcup A(\eta)] .
$$

To see that $\bigcup A(\eta)$ is dense in $A(\xi)$, let $h$ be in $A(\xi)$ and let $\varepsilon>0$ be given. Then $h$ is a uniform limit of polynomials of functions in $\bigcup_{f \in A} A_{f}(\xi)$. Find one within $\varepsilon / 2$ of $h$; call it $p$. Let

$$
p=p_{1} p_{2} p_{3}+\cdots+\cdots p_{n}, \quad p_{k} \in A_{f_{k}}(\xi)
$$

Let $M$ be the maximum of the $\left\|p_{j}\right\|$ 's and let $m$ be the maximum number of factors in each term. For each $k$ we can choose an $\eta_{k}<\xi$ and an $h_{k}$ in $A_{f_{k}}\left(\eta_{k}\right)$ within

$$
\delta=\varepsilon / 2 n(M+1)^{m-1}
$$

of $p_{k}$. Put $p^{\prime}=h_{1} h_{2} h_{3}+\cdots+\cdots h_{n}$. If $\varepsilon<1$, then an easy calculation shows that $\left\|p-p^{\prime}\right\| \leqq \varepsilon / 2$. Hence if $\eta$ is the maximum of the $\eta_{k}$ 's, then $p^{\prime}$ is in $A(\eta)$ and $\left\|p^{\prime}-h\right\| \leqq \varepsilon$.

Case 2. $\xi$ is any ordinal less than $\nu$.

We are to prove that $A(\xi+1)$ is a.p. over $A(\xi)$. Since the functions a.p. over a distal algebra form a distal algebra (Proposition 4.1), it is enough to show that each function in a generating set of $A(\xi+1)$ is a.p. over $A(\xi)$. In particular, it suffices to show that $h \in A_{f}(\xi+1)$ implies $h$ a.p. over $A(\xi)$. But $h$ is a.p. over $A_{f}(\xi)$ and hence it is certainly a.p. over any larger distal algebra since it has less conditions to satisfy. Thus $h$ is a.p. over $A(\xi)$, and the proof of the lemma is complete.

We are going to use Furstenberg's main theorem in [8]. Furstenberg considers only separable distal flows and makes the following definition: A separable distal flow $(X, G)$ with a dense orbit is an isometric extension of a separable distal subflow $(Y, G)=\pi(X, G)$ if there is a real-valued function $\rho$ defined on the set

$$
\Delta=\left\{\left(x_{1}, x_{2}\right) \in X \times X \mid \pi\left(x_{1}\right)=\pi\left(x_{2}\right)\right\}
$$

such that

(a) Restricted to the product of any fiber by itself, $\rho$ is a metric for the fiber.

(b) $\rho$ is continuous on $\Delta$.

(c) $\rho\left(g x_{1}, g x_{2}\right)=\rho\left(x_{1}, x_{2}\right)$ for all $g$ in $G$ and for all $\left(x_{1}, x_{2}\right)$ in $\Delta$.

The theorem that Furstenberg proves is this: If $G$ is a locally compact $\sigma$-compact group and if $(X, G)$ is a separable distal flow with a dense orbit, then for some ordinal $\nu$ there is a system $\left\{\left(X_{\xi}, G\right)\right\}$ of subflows indexed on the ordinals $0 \leqq \xi \leqq \nu$ such that

(a) $\left(X_{0}, G\right)$ is the one-point flow, and $\left(X_{v}, G\right)=(X, G)$.

(b) If $\xi \leqq \xi^{\prime}$, then $\left(X_{\xi}, G\right)$ is a subflow of $\left(X_{\xi^{\prime}}, G\right)$, and all the projection mappings are consistent.

(c) If $\xi<\nu$, then $\left(X_{\xi+1}, G\right)$ is an isometric extension of $\left(X_{\xi}, G\right)$.

(d) If $\xi$ is a limit ordinal $\leqq \nu$ and if $x_{1}$ and $x_{2}$ are two distinct points of $X_{\xi}$, then there is some $\eta<\xi$ such that the projection $\pi$ of $X_{\xi}$ to $X_{\eta}$ satisfies $\pi\left(x_{1}\right) \neq \pi\left(x_{2}\right)$. 
LEMMA 4.8. If $A$ and $B$ are distal algebras and if $M(A)$ is an isometric extension of $M(B)$, then $A$ is a.p. over $B$.

Remarks. The proof of this lemma does not require that $A$ be separable; this observation will be of importance in $\S 8$. As a converse to this lemma, it is true that if $A$ and $B$ are separable distal algebras with $A$ a.p. over $B$, then $M(A)$ is an isometric extension of $M(B)$. This fact will not be needed, and its proof is omitted.

Proof. We shall apply Proposition 4.4. Let $t_{n}, t, s_{n}, s$, and $x$ be given as in that proposition, and let $\rho$ define the isometric extension. We first note that if $\pi\left(x_{1}\right)$ $=\pi\left(x_{2}\right)$ and if $u$ is in the Ellis group $\bar{G}$ of $A$, then $\rho\left(u x_{1}, u x_{2}\right)=\rho\left(x_{1}, x_{2}\right)$ by properties (c) and (b) of $\rho$. Since $\pi\left(s_{n} x\right)=\pi(x)=\pi(s x)$, we see that $\rho$ is defined on $\left(t_{n} s_{n} x, t_{n} s x\right)$ and satisfies

$$
\rho\left(t_{n} s_{n} x, t_{n} s x\right)=\rho\left(s_{n} x, s x\right) .
$$

Since $s_{n} \rightarrow s$, the right side tends to zero with $n$. Therefore

$$
\rho\left(t_{n} s_{n} x, t_{n} s x\right) \rightarrow 0 .
$$

To prove the convergence of $t_{n} s_{n} x$, the compactness of $M(A)$ implies that it suffices to prove that any convergent subnet of $t_{n} s_{n} x$ has limit tsx. Suppose a convergent subnet has limit $x_{0}$. Then

$$
\pi\left(x_{0}\right)=\lim \pi\left(t_{n} s_{n} x\right)=\lim \pi\left(t_{n} s x\right)=\pi(t s x)
$$

so that $\rho\left(x_{0}, t s x\right)$ is defined. By the continuity of $\rho$ and the convergence of $t_{n} s x$ to $t s x$, we must have $\rho\left(x_{0}, t s x\right)=0$. Therefore $x_{0}=t s x$, i.e., $t_{n} s_{n} x \rightarrow t s x$.

Proof of Theorem 1.1. In view of Lemma 4.7 it suffices to prove the theorem for the algebra $A_{f}$. The map $g \rightarrow f_{g}$ of $G$ into LUC is a continuous map of a $\sigma$-compact space into a metric space, and the image must be $\sigma$-compact and hence have a countable dense set. Any such dense set of left translates of $f$, together with their conjugates and 1 , generates $A_{f}$. Hence $A_{f}$ is separable. Therefore $M\left(A_{f}\right)$ is separable, and we can apply Furstenberg's theorem to it. Let the flows obtained be $X_{\xi}, \xi \leqq \nu$, let $e$ be the evaluation-at-e element of $X_{v}$, and let $\pi_{\xi}$ be the projection of $X_{v}$ to $X_{\xi}$. Let $A_{\xi}$ be the distal algebra with maximal ideal space $X_{\xi}$ and evaluation-at- $e$ element $\pi_{\xi}(e)$. Conclusions (a) and (b) of Theorem 1.1 are then clear, and conclusion (c) follows from Lemma 4.8. For conclusion (d), let $\xi$ be a limit ordinal $\leqq \nu$. We are to prove that

$$
A_{\xi}=\text { unif. clos. }\left(\bigcup_{\eta<\xi} A_{\eta}\right) \text {. }
$$

The containment $\supseteq$ is evident since $A_{\xi} \supseteq A_{\eta}$ for each $\eta$. In the reverse direction consider the members of $\bigcup A_{\eta}$ as continuous functions on $X_{\xi}$. These functions form an algebra containing the constants and closed under conjugation; we claim this algebra separates points of $X_{\xi}$. In fact, let $x_{1}$ and $x_{2}$ be points of $X_{\xi}$. Find $\eta$ such that the projection $\pi$ of $X_{\xi}$ to $X_{\eta}$ satisfies $\pi\left(x_{1}\right) \neq \pi\left(x_{2}\right)$, find $f$ in $A_{\eta}$ which 
separates $\pi\left(x_{1}\right)$ and $\pi\left(x_{2}\right)$, and lift $f$ to $X_{\xi}$. Then the lifted $f$ separates $x_{1}$ and $x_{2}$. By the Stone-Weierstrass Theorem, $A_{\xi}=\operatorname{cl}\left(\bigcup A_{\eta}\right)$.

5. Fourier analysis of almost periodic extensions. In this section we begin the proof of Theorem 1.2. The order of what we shall do is this: We first define a convolution, an invariant mean, and the projection operators $P_{\lambda}$. In $\S 6$ we prove the "finite-dimensionality" of the analysis, the orthogonality relation, Parseval's equality, and the approximation theorem. The problem of irreducibility and equivalence will be treated in $\S 7$.

Throughout this section we shall use the following notation: $A$ and $B$ are distal algebras with $A$ a.p. over $B, \pi$ is the projection of $M(A)$ onto $M(B), \bar{G}$ is the Ellis group of $M(A), e$ is the identity of $G$ or the evaluation-ate element of $M(A)$ or $M(B), X_{y}$ is the fiber $\pi^{-1}(y)$ in $M(A)$, and $I_{y}$ is the compact group defined in $\S 4$.

LEMMA 5.1. If $t_{n}$ and $t$ are in $\bar{G}$ with $t_{n} \rightarrow t$ and if $f$ is continuous on $M(A)$, then, for any $y$ in $M(B), f\left(t_{n} x\right)$ converges to $f(t x)$ uniformly for $x$ in the fiber $X_{y}$.

Proof. Let $\varepsilon>0$ be given and fix a point $x_{0}$ in $X_{y}$. Let $s$ and $s_{0}$ denote elements of $I_{y}$. By Proposition 4.4, $t s\left(x_{0}\right)$ is jointly continuous in $s$ and in $t$, where $t$ ranges through $\bar{G}$. This joint continuity, together with the continuity of $f$, means that for each $s_{0}$

$$
\left|f\left(t^{\prime} s x_{0}\right)-f\left(t s_{0} x_{0}\right)\right|<\varepsilon / 2
$$

for all $s$ in some open neighborhood $N\left(s_{0}\right)$ of $s_{0}$ and for all $t^{\prime}$ in some neighborhood of $t$. Then $\left|f\left(t_{n} s x_{0}\right)-f\left(t s^{\prime} x_{0}\right)\right|<\varepsilon$ for $s$ and $s^{\prime}$ in $N\left(s_{0}\right)$ as long as $n \geqq n\left(s_{0}\right)$. In particular,

$$
\left|f\left(t_{n} s x_{0}\right)-f\left(t s x_{0}\right)\right|<\varepsilon
$$

for $s$ in $N\left(s_{0}\right)$ and $n \geqq n\left(s_{0}\right)$. The sets $N\left(s_{0}\right)$, as $s_{0}$ varies, cover $I_{y}$, and a finite number suffices. We therefore obtain

$$
\left|f\left(t_{n} s x_{0}\right)-f\left(t s x_{0}\right)\right|<\varepsilon
$$

for all $s$ in $I_{y}$ if $n$ is sufficiently large. But $I_{y}$ is transitive on $X_{y}$ by Corollary 4.5, and hence $\left|f\left(t_{n} x\right)-f(t x)\right|<\varepsilon$ for all $x$ in $X_{y}$ if $n$ is sufficiently large.

Proposition 5.2. Let $p(s)$ be any bounded Borel function on $I_{e}$ which is constant on conjugate classes. If $f$ is any continuous function on $M(A)$, then the equality

$$
h(x)=\int_{s \in I_{e}} p(s) f\left(t s t^{-1} x\right) d m_{e}(s), \quad \pi(t e)=\pi(x),
$$

where $m_{e}$ is normalized Haar measure on $I_{e}$, unambiguously defines a continuous function $h$ on $M(A)$.

Remark. The function $h$ of the proposition will be denoted $f \times p$. This function may be thought of as a convolution if $p(s)$ is replaced by $p\left(s^{-1}\right)$.

Proof. We first show that the definition is unambiguous, i.e., that it does not depend on the choice of $t$. Let $\pi\left(t^{\prime} e\right)=\pi(x)=\pi(t e)$. Then the restriction of $t^{-1} t^{\prime}$ to 
$X_{e}$ is a member of $I_{e}$. Using in succession the facts that $m_{e}$ is invariant on both sides and that $p$ is unchanged by inner automorphisms, we find

$$
\begin{aligned}
\int_{s \in I_{e}} p(s) f & \left.t s t^{-1} x\right) d m_{e}(s) \\
= & \int_{s \in I_{e}} p\left[\left(t^{-1} t^{\prime}\right) s\left(t^{-1} t^{\prime}\right)^{-1}\right] f\left[t\left(t^{-1} t^{\prime}\right) s\left(t^{-1} t^{\prime}\right)^{-1} t^{-1} x\right] d m_{e}(s) \\
= & \int_{s \in I_{e}} p(s) f\left(t^{\prime} s\left(t^{\prime}\right)^{-1} x\right) d m_{e}(s) .
\end{aligned}
$$

Therefore the definition is unambiguous.

Next we show that $h$ is continuous. Let $x_{n} \rightarrow x$ and let $x_{n}=t_{n} e$. We are to show that $h\left(x_{n}\right) \rightarrow h(x)$. If not, choose a subnet of $x_{n}$ so that $h\left(x_{n}\right)$ converges to something else, and choose a further subnet so that $t_{n}$ converges, say to $t$. Then $t e=x$. Apply Lemma 5.1 to the fiber $X_{e}$. Then for $n$ sufficiently large

$$
\left|f\left(t_{n} z\right)-f(t z)\right| \leqq \varepsilon
$$

for all $z$ in $X_{e}$. If $s$ is in $I_{e}$, then $s t_{n}^{-1} x_{n}$ is in $X_{e}$ and hence

$$
\left|f\left(t_{n} s t_{n}^{-1} x_{n}\right)-f\left(t s t_{n}^{-1} x_{n}\right)\right| \leqq \varepsilon
$$

for all $s$ in $I_{e}$ as long as $n$ is sufficiently large. But $t_{n}^{-1} x_{n}=e=t^{-1} x$, and thus

$$
\left|f\left(t_{n} s t_{n}^{-1} x_{n}\right)-f\left(t s t^{-1} x\right)\right| \leqq \varepsilon
$$

for large $n$. For such $n$ we evidently have

$$
\left|h\left(x_{n}\right)-h(x)\right| \leqq \varepsilon\|p\|_{\infty} .
$$

Therefore $h$ is continuous.

LEMMA 5.3. If $f$ is a continuous function on $M(A)$ and if $p$ and $q$ are bounded Borel functions on $I_{e}$ which are constant on conjugate classes, then

$$
(f \times p) \times q=(f \times q) \times p .
$$

Proof. We have

$$
\begin{aligned}
(f \times p) \times q(x) & =\int_{s} q(s)(f \times p)\left(t s t^{-1} x\right) d s, \quad \pi(t e)=\pi(x) \\
& =\int_{s} \int_{r} q(s) p(r) f\left[\left(t r t^{-1}\right)\left(t s t^{-1} x\right)\right] d r d s \\
& =\int_{s} \int_{r} q(s) p(r) f\left(t r s t^{-1} x\right) d r d s .
\end{aligned}
$$

Similarly

$$
(f \times q) \times p(x)=\int_{r} \int_{s} q(s) p(r) f\left(t s r t^{-1} x\right) d s d r .
$$

Under the change of variable $s \rightarrow r s r^{-1}$, this last expression is equal to

$$
\int_{r} \int_{s} q\left(r s r^{-1}\right) p(r) f\left(t r s t^{-1} x\right) d s d r
$$

Since $q\left(r s r^{-1}\right)=q(s)$, the proof is completed by applying Fubini's Theorem. 
With the aid of this convolution, we introduce the functional that will play the role of an invariant mean. If $p$ is taken to be identically one on $I_{e}$, then $f \times 1$ is a constant function on each fiber. In fact, if $\pi(x)=\pi\left(x^{\prime}\right)$, let $r x=x^{\prime}$ for some $r$ in $I_{\pi(x)}$. Then

$$
\begin{aligned}
f \times 1\left(x^{\prime}\right) & =\int_{s} f\left(t s t^{-1} x^{\prime}\right) d s, \quad \pi(t e)=\pi\left(x^{\prime}\right) \\
& =\int_{s} f\left((r t) s(r t)^{-1} x^{\prime}\right) d s \quad \text { by unambiguity } \\
& =\int_{s} f\left((r t) s t^{-1} x\right) d s \\
& =\int_{s} f\left(t s t^{-1} x\right) d s \quad \text { under } s \rightarrow\left(t^{-1} r^{-1} t\right) s \\
& =f \times 1(x) .
\end{aligned}
$$

We can therefore define unambiguously a function on $M(B)$ by

$$
E(f \mid B)(y)=f \times 1(x), \quad \pi(x)=y .
$$

By Proposition 5.2, $E(f \mid B)$ is continuous. The explanation of notation independent of $A$ appears in the remark with Proposition 5.5.

LemmA 5.4. If $t$ is in $\bar{G}$, then the map $\phi_{t}$ defined on $I_{e}$ by $\phi_{t}(s)=t s t^{-1} \mid X_{\pi(t e)}$ is a topological isomorphism of $I_{e}$ onto $I_{\pi(t))}$. If $t^{\prime}$ also is in $\bar{G}$ and if $\pi(t e)=\pi\left(t^{\prime} e\right)$, then $\phi_{t}$ and $\phi_{t}$, differ by an inner automorphism.

Proof. Since $\pi\left(t s t^{-1}(t e)\right)=\pi(t e)$, Lemma 4.3 shows that $t s t^{-1} \mid X_{\pi(t e)}$ is in $I_{\pi(t e)}$. The map $\phi_{t}$ is clearly a homomorphism, and it is one-one onto because $s^{\prime} \rightarrow t^{-1} s^{\prime} t$ is a two-sided inverse. Moreover $\phi_{t}$ is continuous because it is the composition of the continuous maps $s \rightarrow t s$ (Proposition 4.4) and $t s \rightarrow t s t^{-1}$. Since $I_{e}$ is compact and $I_{\pi(t e)}$ is Hausdorff, $\phi_{t}$ is a homeomorphism.

If $t^{\prime}$ is given, then $t^{\prime} t^{-1}(t e)=t e$ and hence $t^{\prime} t^{-1} \mid X_{\pi}(t e)$ is in $I_{\pi}(t e)$. Since

$$
t^{\prime} t^{-1} \phi_{t}(s)\left(t^{\prime} t^{-1}\right)^{-1}=\phi_{t^{\prime}}(s) \text {, }
$$

$\phi_{t}$ and $\phi_{t^{\prime}}$ differ by an inner automorphism. The proof is complete.

The map $\phi_{t}$ of Lemma 5.4, being a topological isomorphism, carries normalized Haar measure $m_{e}$ of $I_{e}$ over to normalized Haar measure $m_{y}$ of $I_{y}$, and there is thus an alternate way of defining $E(f \mid B)$, namely

$$
E(f \mid B)=\int_{r \in I_{y}} f(r x) d m_{y}(r), \quad \pi(x)=y .
$$

That is, $E(f \mid B)(y)$ is the integral over $X_{y}$ of $f$ with respect to the unique normalized left $I_{y}$-invariant measure on $X_{y}$. To see that these definitions are equivalent, let 
$\pi(t e)=\pi(x)$ and observe that

$$
\begin{aligned}
\int_{r \in I_{y}} f(r x) d m_{y}(r) & =\int_{r \in t I_{e} t^{-1}} f(r x) d m_{y}(r) \\
& =\int_{s \in I_{e}} f\left(t s t^{-1} x\right) d m_{y}\left(t s t^{-1}\right) \\
& =\int_{s \in I_{e}} f\left(t s t^{-1} x\right) d m_{e}(s) .
\end{aligned}
$$

Proposition 5.5. $E(\cdot \mid B)$ has these properties:

(1) $E(f+h \mid B)=E(f \mid B)+E(h \mid B)$.

(2) $E(b f \mid B)=b E(f \mid B)$ for $b$ in $B$.

(3) $E(1 \mid B)=1$.

(4) If $f \geqq 0$, then $E(f \mid B) \geqq 0$.

(5) $E(\bar{f} \mid B)=[\overline{E(f \mid B)}]$.

(6) $E\left(f_{g} \mid B\right)=E(f \mid B)_{g}$ for $g$ in $G$.

(7) If $f$ and $h$ agree on a fiber $X_{y}$, then

$$
E(f \mid B)(y)=E(h \mid B)(y) .
$$

REMARK. It is shown in [10] that these seven properties determine $E(\cdot \mid B)$ uniquely. It follows that $E(f \mid B)$ does not depend upon $A$ as long as $f \in A$ and $B \subseteq A \subseteq A P(B)$.

Proof. In view of the alternate definition of $E(\cdot \mid B)$ above, all of the properties, except possibly (6), are obvious. For (6) we have

$$
\begin{aligned}
E\left(f_{g} \mid B\right)(y) & =f_{g} \times 1(x), \quad \pi(x)=y \\
& =\int_{s} f_{g}\left(t s t^{-1} x\right) d s, \quad \pi(t e)=\pi(x) \\
& =\int_{s} f\left(g t s t^{-1} x\right) d s \\
& =\int_{s} f\left((g t) s(g t)^{-1} g x\right) d s, \quad \pi(g t e)=\pi(g x) \\
& =f \times 1(g x) \\
& =E(f \mid B)_{g}(y) .
\end{aligned}
$$

We introduce the projection operators $P_{\lambda}$ of Theorem 1.2. Let $\Lambda=\{\lambda\}$ be the set of equivalence classes of irreducible (finite-dimensional) continuous unitary representations of the compact group $I_{e}$. Let $\chi_{\lambda}(s), s$ in $I_{e}$, be the trace of $\lambda$ and let $d_{\lambda}$ be the degree of $\lambda$. The map $\phi_{t}$ defined in Lemma 5.4 is a topological isomorphism of $I_{e}$ onto $I_{\pi(t e)}$ and it therefore induces a map of the set of representations of the one group onto the set for the other. If $\pi(t e)=\pi\left(t^{\prime} e\right)$, then the facts that $\phi_{t}$ and $\phi_{t^{\prime}}$ differ by an inner automorphism and that equivalence classes of representations are invariant under conjugation imply that the maps of representations induced by $\phi_{t}$ and $\phi_{t^{\prime}}$ are identical. Thus for fixed $\lambda$, we can speak umambiguously of $\chi_{\lambda}$ as associated to all $I_{y}$. 
Define $P_{\lambda} f$ for $f$ continuous on $M(A)$ by

$$
P_{\lambda} f(x)=d_{\lambda} \int_{r \in I_{y}} \overline{\chi_{\lambda}(r)} f(r x) d m_{y}(r), \quad \pi(x)=y,
$$

where $m_{y}$ is normalized Haar measure on $I_{y}$. Restricted to any fiber $X_{y}, P_{\lambda} f$ is the $L^{2}$-projection of $f$ to the finite-dimensional $I_{y}$-invariant subspace associated with the representation $\lambda$.

Lemma 5.6. If $f$ is continuous on $M(A)$, then $P_{\lambda} f=f \times d_{\lambda} \bar{\chi}_{\lambda}$. Consequently $P_{\lambda} f$ is continuous on $M(A)$.

Proof.

$$
\begin{aligned}
P_{\lambda} f(x) & =d_{\lambda} \int_{r \in I_{y}} \overline{\chi_{\lambda, y}(r)} f(r x) d m_{y}(r), \quad y=\pi(t x) \\
& =d_{\lambda} \int_{r \in t I_{e} t^{-1}} \overline{\chi_{\lambda, y}(r)} f(r x) d m_{y}(r) \\
& =d_{\lambda} \int_{s \in I_{e}} \overline{\chi_{\lambda, y}\left(t s t^{-1}\right)} f\left(t s t^{-1} x\right) d m_{y}\left(t s t^{-1}\right) \\
& =d_{\lambda} \int_{s \in I_{e}} \overline{\chi_{\lambda}(s)} f\left(t s t^{-1} x\right) d m_{e}(s) \\
& =f \times d_{\lambda} \bar{\chi}_{\lambda}(x) .
\end{aligned}
$$

Continuity is by Proposition 5.2.

6. Properties of the projection operators. This section consists of proofs of parts (a) through (d) of Theorem 1.2. We retain all of the notation in force in $\S 5$. Notice that the Peter-Weyl Theorem, together with the interpretation of $E(\cdot \mid B)$, shows immediately that the $P_{\lambda}$ are projections and that (b) holds in Theorem 1.2. It is clear also that $P_{\lambda}$ is $B$-linear and hence that image $\left(P_{\lambda}\right)$ is a $B$-submodule of $A$.

Let $U$ be an open set in $M(B)$. Since $E(\cdot \mid B)$ is defined fiber-by-fiber, there is a natural way of defining $E(h \mid B)(y)$ whenever $y$ is in $U$ and $h$ is a continuous function defined on $\pi^{-1}(U)$. That the function $E(h \mid B)(y)$ is continuous for $y$ in $U$ is seen as follows: Fix $y$ and choose an open set $V$ such that $y \in V$ and $\bar{V} \subseteq U$. Apply the Tietze Extension Theorem to extend $h \mid \pi^{-1}(\bar{V})$ to all of $M(A)$, apply $E(\cdot \mid B)$ to the result, and get a continuous function. This continuous function agrees with $E(h \mid B$, in the neighborhood $V$ of $y$.

The proofs of the next two lemmas use the notation $(f, h)=E(f \bar{h} \mid B)$.

LEMMA 6.1. If $\left\{f_{1}, \ldots, f_{n}\right\}$ is a set of continuous functions on $M(A)$ whose restrictions to a single fiber $X_{y}$ are independent, then $f_{1}, \ldots, f_{n}$ are independent on all fibers in some open neighborhood of $y$.

Proof. Form the Gram matrix whose $i-j$ th entry is $\left(f_{i}, f_{j}\right)$. The determinant of this matrix is a continuous function on $M(B)$, and its vanishing at $z$ is equivalent 
with the linear dependence of $\left\{f_{1}, \ldots, f_{n}\right\}$ on $X_{z}$. Since the determinant does not vanish at $y$, there is an open neighborhood of $y$ in which it does not vanish.

LEMMA 6.2. If $\left\{f_{1}, \ldots, f_{n}\right\}$ is a set of continuous functions on $M(A)$ whose restrictions to the fiber $X_{y}$ are independent for every $y$ in a nonempty open set $U$ of $M(B)$ and if $f$ is a continuous function on $M(A)$ which satisfies

$$
f(x)=c_{1}(y) f_{1}(x)+\cdots+c_{n}(y) f_{n}(x), \quad \pi(x)=y,
$$

for all $y$ in $U$, then $c_{1}(y), \ldots, c_{n}(y)$ are continuous on $U$.

Proof. By symmetry it suffices to prove that $c_{n}$ is continuous. Restrict the functions $f_{1}, \ldots, f_{n}$ to $\pi^{-1}(U)$ but give the restrictions the same names. Apply the Gram-Schmidt process fiber-by-fiber to the restrictions, using the inner product formed from $E(\cdot \mid B)$. We obtain thereby functions $\phi_{1}, \ldots, \phi_{n}$ defined on $\pi^{-1}(U)$ whose restrictions to each fiber are orthonormal and which satisfy

$$
\begin{gathered}
\phi_{1}=f_{1} / E\left(\left|f_{1}\right|^{2} \mid B\right), \\
h_{k}=f_{k}-\left(f_{k}, \phi_{1}\right) \phi_{1}-\cdots-\left(f_{k}, \phi_{k-1}\right) \phi_{k-1}, \quad k \geqq 2, \\
\phi_{k}=h_{k} / E\left(\left|h_{k}\right|^{2} \mid B\right)^{1 / 2}, \quad k \geqq 2 .
\end{gathered}
$$

The continuity of $E(\cdot \mid B)$ ensures that the $\phi_{k}$ are continuous on $\pi^{-1}(U)$. On one hand we have

$$
\begin{aligned}
f(x) & =\sum_{k=1}^{n} c_{k}(y) f_{k}(x) \\
& =c_{n}(y) E\left(\left|h_{n}\right|^{2} \mid B\right)(y)^{1 / 2} \phi_{n}+\sum_{k=1}^{n-1}(-) \phi_{k},
\end{aligned}
$$

and on the other hand we have

$$
f(x)=\sum_{k=1}^{n}\left(f, \phi_{k}\right) \phi_{k}
$$

Since $E\left(\left|h_{n}\right|^{2} \mid B\right)$ is nowhere zero on $U$, we conclude

$$
c_{n}=\left(f, \phi_{n}\right) / E\left(\left|h_{n}\right|^{2} \mid B\right)^{1 / 2},
$$

and $c_{n}$ is therefore continuous. The proof is complete.

A $B$-submodule $M$ of $A$ will be said to be of constant (finite) dimension $n$ if, for each $y$ in $M(B)$, the restrictions of members of $M$ to $X_{y}$ form a complex vector space of dimension $n$.

LemMA 6.3. If $M$ is a G-invariant $B$-submodule of $A$ such that, for some $y$ in $M(B)$, the restrictions of members of $M$ to $X_{y}$ form a finite-dimensional vector space, then $M$ is of constant dimension.

Proof. Fix an integer $n$, and let $U$ be the set of points $y$ in $M(B)$ such that the vector space of restrictions to $X_{y}$ has dimension $\geqq n$. It suffices to prove that either $U=\varnothing$ or $U=M(B)$. Thus let $y$ be in $U$, and let $f_{1}, \ldots, f_{n}$ be members of $M$ whose 
restrictions to $X_{y}$ are independent. By Lemma 6.1, the restrictions to neighboring fibers are also independent. Therefore $U$ contains a neighborhood of $y$ and $U$ is open. We show $U$ is $G$-invariant. Let $y$ be in $U$, and let $f_{1}, \ldots, f_{n}$ be as above. Then $\left(f_{1}\right)_{g^{-1}}, \ldots,\left(f_{n}\right)_{g^{-1}}$ are independent on the fiber $X_{g y}$ and hence $g y$ is in $U$. It follows that the complement of $U$ is a closed $G$-invariant subset of $M(B)$, and by Corollary 3.3 we see that such a set must be $\varnothing$ or $M(B)$.

LEMMA 6.4. If $M$ is a $B$-submodule of $A$ of constant dimension $n$, then $M$ is finitelygenerated as a B-module. Specifically, any set of elements $\left\{f_{i}\right\}$ of $M$ whose restrictions to each $X_{y}$ span a space of dimension $n$ has a finite subset which is a set of generators for $M$.

Proof. Let $\left\{f_{i}\right\}$ be given. For each $y$ in $M(B)$ find $n$ functions from the set which are independent on $X_{y}$ and find, by Lemma 6.1, an open neighborhood of $z$ 's in $M(B)$ such that these $n$ functions continue to be independent on $X_{z}$. As $y$ varies, these neighborhoods cover $M(B)$, and a finite number $N_{1}, \ldots, N_{m}$ suffices. Let the corresponding $y$ 's be $y_{1}, \ldots, y_{m}$ and let the functions be

$$
f_{k}^{j}, \quad \text { where } 1 \leqq j \leqq m \text { and } 1 \leqq k \leqq n .
$$

This set of functions has the same vector-space-spanning property that $\left\{f_{i}\right\}$ has. We shall prove this set generates $M$. In view of the compactness we can choose a partition of unity $\left\{\phi_{j}\right\}$ on $M(B)$ subordinate to the cover $\left\{N_{j}\right\}$ such that each $\phi_{j}$ vanishes on the complement of a compact subset $K_{j}$ of $N_{j}$. Let $h$ in $M$ be given. The only properties of $h$ that we shall use are that it is continuous and that its restriction to each fiber is in the subspace spanned by $f_{1}^{1}, \ldots, f_{n}^{m}$. Let $\psi_{j}$ be a continuous function with range in $[0,1]$ which is one on $K_{j}$ and is zero on the complement of $N_{j}$. By Lemma 6.2 we can write

$$
\psi_{j}(y) h(x)=c_{1}^{j}(y) f_{1}^{j}(x)+\cdots+c_{n}^{j}(y) f_{n}^{j}(x), \quad \pi(x)=y,
$$

with the $c$ 's continuous on $N_{j}$. Then since $\phi_{j} \psi_{j}=\phi_{j}$, we have

$$
\phi_{j} h=\left(\phi_{j} c_{1}^{j}\right) f_{1}^{j}+\cdots+\left(\phi_{j} c_{n}^{j}\right) f_{n}^{j},
$$

and the functions $\phi_{j} c_{k}^{j}$ are everywhere continuous. Summing on $j$, we see that $h$ is a $B$-linear sum of $f_{1}^{1}, \ldots, f_{n}^{m}$.

THEOREM 6.5. $P_{\lambda}$ commutes with the left action of $G$. Image $\left(P_{\lambda}\right)$ is left-invariant under $G$, is of constant finite dimension, and is finitely-generated as a B-module.

Proof. For the commutativity with $G$ we have, by Lemma 5.6,

$$
\begin{aligned}
P_{\lambda} f_{g}(x) & =d_{\lambda} \int \overline{\chi_{\lambda}(s)} f_{g}\left(t s t^{-1} x\right) d s, \quad \pi(t e)=\pi(x) \\
& =d_{\lambda} \int \overline{\chi_{\lambda}(s)} f\left((g t) s(g t)^{-1} g x\right) d s, \quad \pi(g t e)=\pi(g x) \\
& =P_{\lambda} f(g x) \\
& =\left(P_{\lambda} f\right)_{g}(x) .
\end{aligned}
$$


Consequently image $\left(P_{\lambda}\right)$ is left-invariant. The restrictions to $X_{e}$ of functions in image $\left(P_{\lambda}\right)$ form a finite-dimensional vector space, by a classical theorem on group representations. By Lemma 6.3, image $\left(P_{\lambda}\right)$ is of constant finite dimension, and by Lemma 6.4 it is finitely-generated as a $B$-module.

LemMa 6.6. If $X$ is a compact Hausdorff space and if $\left\{f_{\alpha} \mid \alpha \in P\right\}$ is a nonempty (and possibly uncountable) set of nonnegative continuous functions whose pointwise sum

$$
f(x)=\sum_{\alpha} f_{\alpha}(x)
$$

is everywhere finite and continuous, then all but a countable number of the functions are identically zero.

Proof. For fixed $n$, the sets

$$
\left\{x \mid f(x)-\sum_{\alpha \in F} f_{\alpha}(x)<1 / n\right\}
$$

form an open cover of $X$ as $F$ varies through the finite subsets of $P$. By compactness there is a finite subcover, and, if we form the union $F_{n}$ of the corresponding finite subsets of $P$, then we have $\left\|f-\sum_{\alpha \in F_{n}} f_{\alpha}\right\|<1 / n$. It follows that $f=\sum_{\alpha \in} \cup_{F_{n}} f_{\alpha}$ and hence that all but countably many of the functions $f_{\alpha}$ are identically 0 .

THEOREM 6.7. If $f$ is continuous on $M(A)$, then $P_{\lambda} f$ is identically zero for all but countably many $\lambda$, and

$$
E\left(|f|^{2} \mid B\right)=\sum_{\lambda} E\left(\left|P_{\lambda} f\right|^{2} \mid B\right),
$$

the convergence of the right side being uniform on $M(B)$.

Proof. The equality (6.1) pointwise is an immediate consequence of the PeterWeyl Theorem and the interpretation of $E(\cdot \mid B)$. By Lemma 6.6, $E\left(\left|P_{\lambda} f\right|^{2} \mid B\right)=0$ for all but countably many $\lambda$. Since $\left|P_{\lambda} f\right|^{2}$ is continuous and nonnegative on each fiber, it must be zero for the same $\lambda$ 's. Finally the statement of the uniformity of the convergence follows from Dini's Theorem.

LEMMA 6.8. If $f$ is continuous on $M(A)$, then for any $\varepsilon>0$ there is a neighborhood $N$ of the identity in $I_{e}$ such that, for all $x$ in $M(A)$ and for all $t$ in $\bar{G}$ such that $\pi(t e)$ $=\pi(x)$, the inequality

$$
\left|f\left(t s t^{-1} x\right)-f(x)\right| \leqq \varepsilon
$$

holds whenever $s \in N$.

Proof. The expression under consideration is equal to

$$
\left|f\left(t s t^{-1} x\right)-f\left(t t^{-1} x\right)\right|
$$

and $t^{-1} x$ is in the fiber $X_{e}$. Thus it is sufficient to prove that

$$
\mid f(\text { tsre })-f(\text { tre }) \mid \leqq \varepsilon
$$


for all $t$, for all $r$ in $I_{e}$, and for all $s$ in a neighborhood of the identity in $I_{e}$. By joint continuity of $f(t s r e)$ in the three variables $t, s$, and $r$, for each $t_{0}$ and $r_{0}$ there is a product open neighborhood of $t$ 's, $s$ 's, and $r$ 's, where the $s$ 's form a neighborhood of the identity, such that

$$
\left|f(t s r e)-f\left(t_{0} r_{0} e\right)\right|<\varepsilon / 2
$$

and hence

$$
\mid f(\text { tsre })-f(\text { tre }) \mid<\varepsilon .
$$

As $t_{0}$ and $r_{0}$ vary, these product neighborhoods cover the product of the $t$-space and the $r$-space, and there is therefore a finite subcover. Then (6.2) holds for $s$ in a neighborhood depending on what subcover set $(t, r)$ is in. Intersecting the corresponding finitely many $s$-neighborhoods of the identity to obtain a neighborhood $N$, we see that (6.2) holds for all $t$ and $r$ as long as $s$ is in $N$.

LEMMA 6.9. If $K$ is a compact group and if $U$ is a neighborhood of $e$, then there is a continuous function $p \geqq 0$ on $K$ such that

(1) $p$ vanishes outside $U$,

(2) $\int p(s) d s=1$,

(3) $p$ is constant on conjugate classes.

Proof. Choose a family of two-sided invariant semimetrics $\left\{d_{m}\right\}$ for $K$ which generate the topology of $K$. Since $U$ is open, we can choose finitely many, say $d_{1}, \ldots, d_{n}$, and a set of $\varepsilon$ 's, say $\varepsilon_{1}, \ldots, \varepsilon_{n}$, such that

$$
V=\left\{s \mid d_{j}(e, s)<\varepsilon_{j} \quad \text { for } j=1, \ldots, n\right\} \subseteq U .
$$

Since

$$
d_{j}\left(g s g^{-1}, e\right)=d_{j}(g s, e g)=d_{j}\left(s, g^{-1} e g\right)=d_{j}(s, e),
$$

$V$ is closed under the operation of conjugation. Form the quotient space of conjugate classes, and let $\sigma$ be the projection. Then $\sigma(K-V)$ is compact and disjoint from $\sigma(e)$. If we can show the quotient is Hausdorff, then we choose a function $h$ which vanishes on $\sigma(K-V)$, is $\geqq 0$, and is one at $\sigma(e)$. Lifting $h$ to a function $p$ on $K$ and renormalizing, we get the desired result.

In order for the quotient to be Hausdorff, it is necessary and sufficient that the equivalence relation be closed. Let $x_{i} \sim y_{i}, x_{i} \rightarrow x$, and $y_{i} \rightarrow y$. Then $x_{i}=g_{i} y_{i} g_{i}^{-1}$. Choose a convergent subnet of $\left\{g_{i}\right\}$, say with limit $g$. By joint continuity $x=g y g^{-1}$. Hence $x \sim y$.

THEOREM 6.10. There is a net of bounded B-linear G-commuting operators $T_{j}$ on $A$ with this property: If $P_{\lambda} f$ is nonzero for $\lambda=1,2,3, \ldots$, then $T_{j} f$ is in the weak direct sum

$$
\operatorname{image}\left(P_{1}\right) \oplus \operatorname{image}\left(P_{2}\right) \oplus \cdots
$$

and $\left\{T_{1} f\right\}$ converges uniformly to $f$. 
Proof. The required net will be a subnet of the following net: The directed set $D$ is the product of the set of finite subsets of $\lambda$ 's by the set of neighborhoods of the identity in $I_{e}$. To each such neighborhood $U$, associate a function $p$ satisfying the conditions of Lemma 6.9 for $K=I_{e}$. The operator that corresponds to $j$ $=\left(\left\{\lambda_{1}, \ldots, \lambda_{n}\right\}, U\right)$ is the composition of convolution by $p$ followed by $P_{\lambda_{1}}+\cdots$ $+P_{\lambda_{n}}$. Lemmas 5.3 and 5.6 show that $T_{j} f$ always lies in the weak direct sum (6.3). The $T_{j}$ 's are clearly bounded and $B$-linear, and they commute with $G$ by the same sort of calculation as in the proof of Theorem 6.5.

We first show that for any $\varepsilon>0$ and functions $f_{1}, \ldots, f_{k}$, we can find arbitrarily large $j$ such that $\left\|f_{i}-T_{j} f_{i}\right\|<\varepsilon$ for $i=1, \ldots, k$. Thus let $j_{0}=\left(\left\{\lambda_{1}, \ldots, \lambda_{n_{0}}\right\}, U\right)$ be given. Choose a neighborhood $N_{i}$ for $\varepsilon$ and $f_{i}$ in accordance with Lemma 6.8, let $N=U \cap N_{1} \cap \cdots \cap N_{k}$, and let $p$ be the function associated to $N$ by Lemma 6.9. Then $\left\|f_{i}-f_{i} \times p\right\| \leqq \varepsilon$ for each $i$ because

$$
\begin{aligned}
\left\|f_{i}-f_{i} \times p\right\| & \leqq \sup _{x} \int_{s}\left|f_{i}\left(t s t^{-1} x\right)-f(x)\right| p(s) d s, \quad \pi(t e)=\pi(x) \\
& \leqq \sup _{x ; s \in N}\left|f\left(t s t^{-1} x\right)-f(x)\right|\left(\int p(r) d r\right), \quad \pi(t e)=\pi(x) \\
& \leqq \varepsilon
\end{aligned}
$$

Put $h_{i}=f_{i}-P_{\lambda_{1}} f_{i}-\cdots-P_{\lambda_{n}} f_{i}$, where $n \geqq n_{0}$ is chosen large enough so that $E\left(\left|h_{i}\right|^{2} \mid B\right)^{1 / 2}$ is uniformly $\leqq \varepsilon /\|p\|_{2}$ for $i=1, \ldots, k$. Such an $n$ exists by Theorem 6.7 since the $P_{\lambda}$ 's are orthogonal. For fixed $x$ apply the Schwarz inequality to $h_{i} \times p(x)$. Then

$$
\begin{aligned}
\left|h_{i} \times p(x)\right| & =\left|\int_{s} h_{i}\left(t s t^{-1} x\right) p(s) d s\right| \\
& \leqq\left(\int_{s}\left|h_{i}\left(t s t^{-1} x\right)\right|^{2} d s\right)^{1 / 2}\left(\int_{s}|p(s)|^{2} d s\right)^{1 / 2} \\
& =\left[E\left(\left|h_{i}\right|^{2} \mid B\right)(\pi(x))\right]^{1 / 2}\|p\|_{2} \\
& \leqq \varepsilon
\end{aligned}
$$

Hence, by Lemma 5.3,

$$
\begin{aligned}
\| f_{i}-\left(P_{\lambda_{1}}\left(f_{i} \times p\right)+\cdots\right. & \left.+P_{\lambda_{n}}\left(f_{i} \times p\right)\right) \| \\
& \leqq\left\|f_{i}-f_{i} \times p\right\|+\left\|f_{i} \times p-\left(\sum P_{\lambda_{m}}\left(f_{i} \times p\right)\right)\right\| \\
& \leqq \varepsilon+\left\|f_{i} \times p-\left(\sum P_{\lambda_{m}} f_{i}\right) \times p\right\| \\
& =\varepsilon+\left\|h_{i} \times p\right\| \\
& \leqq 2 \varepsilon
\end{aligned}
$$

as asserted.

Finally let $E$ be the set of all triples $(d, \varepsilon, F)$ such that $d$ is in the directed set above, $F$ is a finite set of functions in $A$, and $\left\|T_{d} f-f\right\|<\varepsilon$ for all $f$ in $F$. Partially order $E$ by the product ordering, and define $T_{(d, \varepsilon, F)}=T_{d}$. The argument of the 
previous paragraph shows that any triple $(d, \varepsilon, F)$, whether or not satisfying the inequality defining $E$, has members of $E$ beyond it in the product ordering. Hence the result is a subnet. Its convergence is obvious.

7. Irreducibility and equivalence. We continue to use the notation of $\S 6$. This section is occupied with the proof of parts (e) and (f) of Theorem 1.2. A nonzero $G$-invariant $B$-submodule of $A$ is irreducible if it contains no proper nonzero $G$-invariant $B$-submodules. We note some facts connected with the proof of Lemma 6.4.

(1) If $M$ is a $B$-submodule of $A$ of constant dimension $n$, then there is a finite open cover $\left\{U_{1}, \ldots, U_{k}\right\}$ of $M(B)$ and there is a set of functions $\left\{f_{i}^{j} \mid 1 \leqq i \leqq n\right.$, $1 \leqq j \leqq k\}$ such that for each fixed $j$ the functions $f_{i}^{j}, 1 \leqq i \leqq n$, are independent on every fiber in $\pi^{-1}\left(U_{j}\right)$.

(2) Any such set $\left\{f_{i}^{j}\right\}$ generates $M$ as a $B$-module.

(3) If, for each $y$ in $M(B)$, the restrictions of members of $M$ to the fiber $\pi^{-1}(y)$ form a vector space $V_{y}$, then $M$ contains all functions $f$ continuous on $M(A)$ such that, for each $y, f \mid \pi^{-1}(y)$ is in $V_{y}$.

(4) Let $h_{i}^{j}$ be defined and continuous only on $\pi^{-1}\left(U_{j}\right)$ and let $h_{1}^{j}, \ldots, h_{n}^{j}$ be independent on every fiber in $\pi^{-1}\left(U_{j}\right)$. We describe a procedure for getting from these functions a set of generators for $M$. Let $\left\{\phi_{j}\right\}$ be a partition of unity on $M(B)$ such that each $\phi_{j}$ is nonzero only on an open set $W_{j}$ with $\bar{W}_{j} \subseteq U_{j}$. Then the functions

$$
\begin{aligned}
f_{i}^{j}(x) & =\phi_{j}(\pi(x)) h_{i}^{j}(x) \quad \text { if } x \in \pi^{-1}\left(W_{j}\right) \\
& =0 \text { otherwise }
\end{aligned}
$$

are globally defined on $M(A)$ and are continuous. The sets $W_{j} \operatorname{cover} M(B)$, and the functions $f_{1}^{j}, \ldots, f_{n}^{j}$ are independent on every fiber in $\pi^{-1}\left(W_{j}\right)$. By (2) the functions $f_{i}^{j}$ generate $M$.

Any set of functions $\left\{h_{i}^{j}\right\}$ satisfying the properties described in (4) will be called a local base for $M$.

Lemma 7.1. If $M$ is a B-submodule of $A$ of constant dimension and if $\left\{U_{j}\right\}$ is a finite open cover of $M(B)$ which admits a local base for $M$, then there is a local base $h_{i}^{j}$ relative to the same cover such that $E\left(h_{i}^{j} \bar{h}_{k}^{j} \mid B\right)=\delta_{i k}$ for all $i, j$, and $k$.

Proof. The Gram-Schmidt process works for any local base. The continuity on each $\pi^{-1}\left(U_{j}\right)$ of the functions involved is preserved because if $h$ is continuous on $\pi^{-1}(U)$, then $E(h \mid B)$ is continuous on $U$.

The set of functions constructed in Lemma 7.1 will be called a local orthonormal base for $M$.

Lemma 7.2. If $M$ is a B-submodule of $A$ of constant dimension a and if $N$ is a $B$-submodule of $M$ of constant dimension $b$, then relative to some finite open cover $\left\{W_{r}\right\}$ of $M(B)$, there is a local orthonormal base for $N$ which can be extended to a local orthonormal base for $M$. 
Proof. By remark (1) let $\left\{U_{j}\right\}$ be a finite open cover of $M(B)$ relative to which $M$ has a local base, and let $\left\{V_{k}\right\}$ be any finite refinement of $\left\{U_{j}\right\}$ for which $N$ has a local orthonormal base (existence by remark (1) and Lemma 7.1). Let such a base consist of the functions $\left\{n_{i}^{k} \mid 1 \leqq i \leqq b\right\}$. Let, by Lemma 7.1, $\left\{h_{i}^{j}\right\}$ be a local orthonormal base for $M$ relative to $\left\{U_{j}\right\}$. Since $\left\{V_{k}\right\}$ is a refinement of $\left\{U_{j}\right\}$, we may choose a $j(k)$ for each $k$ such that $V_{k} \subseteq U_{j}$. Let $m_{i}^{k}$ be the restriction of $h_{i}^{j(k)}$ to $\pi^{-1}\left(V_{k}\right)$. Then

$$
\left\{m_{i}^{k} \mid 1 \leqq i \leqq a\right\}
$$

is a local orthonormal base for $M$ relative to the cover $\left\{V_{k}\right\}$. Form, for fixed $k$, the $a$ functions

$$
m_{i}^{k}-\sum_{l=1}^{b}\left(m_{i}^{k}, n_{l}^{k}\right) n_{l}^{k}
$$

On each fiber in $\pi^{-1}\left(V_{k}\right)$ these functions span a subspace of dimension $a-b$ which is orthogonal to that spanned by the restrictions of the $n_{l}^{k}$. For each fixed $y$, find $a-b$ of them which are independent, and find an open neighborhood of $y$ in $V_{k}$ in which they remain independent (Lemma 6.1). As $y$ and $k$ vary, these neighborhoods cover $M(B)$ and a finite number suffices. The resulting cover $\left\{W_{r}\right\}$ is a finite refinement of $\left\{V_{k}\right\}$. To each $W_{r}$ choose a $V_{k}$ containing it and choose $y$ that gave rise to $W_{r}$. Retain the $a-b$ functions obtained for $y, V_{k}$, and $W_{r}$. Also retain (and list them first) the restrictions to $\pi^{-1}\left(W_{r}\right)$ of the $b$ functions $n_{i}^{k}, 1 \leqq i \leqq b$. The result is $a$ functions defined on each $\pi^{-1}\left(W_{r}\right)$, and, as $r$ varies, these functions form a local base. Apply the Gram-Schmidt process to obtain a local orthonormal base with the required properties.

Lemma 7.3. Let $M$ be a B-submodule of $A$ of constant dimension $a$ and let $N$ be $a$ $B$-submodule of $M$ of constant dimension $b$. Denote by $N^{\perp}$ the set of all functions $f$ in $M$ such that $E(f \bar{h} \mid B)=0$ for all $h$ in $N$. Then

$$
M=N \oplus N^{\perp} .
$$

Proof. Choose, by Lemma 7.2, a local orthonormal base for $M$ which, for each $U_{j}$, consists of $b$ functions in a local base for $N$ and $a-b$ functions in a local base for $N^{\perp}$. From this local base form a generating set for $M$ according to remark (4). Then each function in the generating set is in $N$ or $N^{\perp}$ and it follows that $M=$ $N+N^{\perp}$. On the other hand, if $f$ is in $N \cap N^{\perp}$, then $E\left(|f|^{2} \mid B\right)=0$ and so $f=0$. Thus $M=N \oplus N^{\perp}$.

Proposition 7.4. If $M$ is a $G$-invariant $B$-submodule of $A$ of constant finite dimension and if $N$ is a $G$-invariant $B$-submodule of $M$, then $M=N \oplus N^{\perp}$ exhibits $M$ as the direct sum of $G$-invariant B-submodules.

Proof. By Lemma 6.3, $N$ is of constant finite dimension; hence, by Lemma 7.3, $M=N \oplus N^{\perp}$. If $f$ is in $N^{\perp}$ and $n$ is in $N$, then $f_{g}$ is in $M$ and

$$
E\left(f_{g} \bar{n} \mid B\right)=E\left(f \bar{n}_{g^{-1}} \mid B\right)_{g}=0 .
$$

Hence $f_{g}$ is in $N^{\perp}$ and $N^{\perp}$ is a $G$-invariant $B$-module such that $M=N \oplus N^{\perp}$. 
THEOREM 7.5. Every G-invariant B-submodule of image $\left(P_{\lambda}\right)$ is finitely-generated, and image $\left(P_{\lambda}\right)$ is the finite orthogonal direct sum of irreducible $G$-invariant $B$-modules.

Proof. A proper $B$-submodule of constant dimension of a $B$-module of constant dimension is of lower dimension. The rest follows from Lemmas 6.3 and 6.4 and Proposition 7.4.

We turn to the problem of equivalence. If $M$ and $N$ are $G$-invariant $B$-submodules of $A$ of constant finite dimension, then $M$ and $N$ are equivalent if there is a one-one bounded $B$-linear transformation $F$ of $M$ onto $N$ which commutes with the action of $G$. (The symmetry of this definition, i.e., that $F^{-1}$ is bounded, follows from the interior mapping theorem and Lemma 7.6 below.) The proof of part (f) of Theorem 1.2 will be an easy matter once we have established the connection between the notion of equivalence above and the usual notion of equivalence under $I_{y}$ of vector spaces of functions on $X_{y}$.

LEMMA 7.6. Any B-submodule $M$ of $A$ of constant finite dimension is complete in the uniform norm.

Proof. Let $\left\{f_{n}\right\}$ be uniformly Cauchy in $M$ and let $f$ be its limit in $A$. Then $f$ is continuous on $M(A)$ and its restriction to any fiber is in the space of restrictions of members of $M$ because this space is finite-dimensional. By remark (3), $f$ is in $M$.

LEMMA 7.7. If $M$ and $N$ are irreducible $G$-invariant $B$-submodules of $A$ of constant finite dimension and if $F$ is a B-linear transformation of $M$ into $N$ which commutes with the action of $G$, then $F$ is zero or $F$ is one-one onto. If, in addition, $F$ is bounded and $M=N$, then $F=c I$ for some complex number $c$.

Proof. Since $\operatorname{kernel}(F)$ and image $(F)$ are $G$-invariant $B$-submodules of $M$ and $N$, respectively, $\operatorname{kernel}(F)=0$ or $M$ and also image $(F)=0$ or $N$. Hence $F$ is zero or one-one onto. If, in addition, $F$ is bounded and $M=N$, then Lemma 4 on p. 567 of [3] and Lemma 7.6 show that the spectrum of $F$ is nonempty. If $c$ is in the spectrum, then $c I-F$ is $B$-linear and commutes with $G$. Since it is not one-one onto, it must be zero.

Proposition 7.8. If $M$ is an irreducible G-invariant B-submodule of $A$ of constant finite dimension, then there is a $\lambda$ such that $M$ is equivalent to an irreducible $B$-submodule of image $\left(P_{\lambda}\right)$.

Proof. Fix $\sigma$ in $\Lambda$ and let image $\left(P_{\sigma}\right)$ decompose as

$$
\operatorname{image}\left(P_{\sigma}\right)=M_{1} \oplus \cdots \oplus M_{m}
$$

with the $M_{i}$ irreducible. Fix $i$, rewrite this expression as

$$
\operatorname{image}\left(P_{\sigma}\right)=M_{i} \oplus\left(M_{1} \oplus \cdots \oplus M_{i-1} \oplus M_{i+1} \oplus \cdots \oplus M_{m}\right),
$$

and let $p_{i}$ be the projection mapping onto $M_{i}$. Since $p_{i}$ is a projection and its domain, kernel, and image are complete (Lemma 7.6), the closed graph theorem shows that 
$p_{i}$ is bounded. Hence $p_{i} P_{\sigma}$ is a bounded $B$-linear $\ldots$.. $M$ into $M_{i}$ which commutes with the action of $G$. By Lemma 7.7, either $M$ is equivalent to $M_{i}$ (and we are done) or else $p_{i} P_{\sigma}(M)=0$. We may thus assume that $p_{i} P_{\sigma}(M)=0$ for all $i$ and $\sigma$. Summing on $i$, we obtain $P_{\sigma}(M)=0$ for all $\sigma$. By Theorem 6.7, $M=0$, a contradiction.

LEMMA 7.9. If $f$ is in $A$, then the function $h$ defined on $M(B)$ as

$$
h(y)=\max _{x \in X_{y}}|f(x)|
$$

is continuous.

REMARK. We shall use the notation $\|f\|_{y}$ in place of $h(y)$ after this lemma.

Proof. Fix $y_{0}$ and let $y_{n} \rightarrow y_{0}$. By compactness we may assume that $h\left(y_{n}\right)$ converges. Choose $x_{n} \in X_{y_{n}}$ so that $h\left(y_{n}\right)=\left|f\left(x_{n}\right)\right|$, and choose a subnet $\left\{x_{n^{\prime}}\right\}$ so that $x_{n^{\prime}}$ converges, say to $x_{0}$. Then

$$
\begin{aligned}
\lim h\left(y_{n}\right) & =\lim \left|f\left(x_{n}\right)\right|=\lim \left|f\left(x_{n^{\prime}}\right)\right|=\left|f\left(x_{0}\right)\right| \leqq \max _{x \in X_{y_{0}}}|f(x)| \\
& =h\left(y_{0}\right) .
\end{aligned}
$$

Failure of equality to hold would mean that $|f(x)|>\left|f\left(x_{0}\right)\right|$ on an open neighborhood $U$ of some point $x_{1}$ in $\pi^{-1}\left(y_{0}\right)$ and that $\pi(U)$ contains no points $y_{n}$ if $n$ is sufficiently large. That is, to complete the proof it suffices to show that $\pi(U)$ contains an open set about $y_{0}$. Let $k$ be a continuous function with values in $[0,1]$ which is 1 at $x_{1}$ and 0 off $U$. Then $E(k \mid B)$, being continuous, is positive in a neighborhood of $y_{0}$, and that neighborhood is contained in $\pi(U)$.

Lemma 7.10. Let $M$ and $N$ be B-submodules of $A$, and iet $F$ be a bounded B-linear map of $M$ into $N$. For each $y$ in $M(B)$, let $U_{y}$ and $V_{y}$ be the vector spaces of restrictions of members of $M$ and $N$, respectively, to $X_{y}$. Then the illentity

$$
F^{y}\left(f \mid X_{y}\right)(x)=F(f)(x) ; \quad f \in M, y \in M(B), x \in X_{y}
$$

consistently defines a linear transformation of $U_{y}$ into $V_{y}$.

Proof. We are to show that if $f_{1}$ and $f_{2}$ agree on $\pi^{-1}(y)$, then so do $F\left(f_{1}\right)$ and $F\left(f_{2}\right)$. Subtract. We are to show that if $f=0$ on $\pi^{-1}(y)$, then $F(f)=0$ on $\pi^{-1}(y)$. Let $\varepsilon>0$ be given. Let, by Lemma 7.9, $N$ be an open neighborhood of $y$ such that $\|f\|_{z} \leqq \varepsilon$ for $z$ in $N$. Choose a continuous function $b$ on $M(B)$ with values in [0. 1? which is 1 at $y$ and 0 off $N$. Then $\|b f\| \leqq \varepsilon$. Hence

$$
\|b F(f)\|=\|F(b f)\| \leqq \varepsilon\|F\|
$$

and $\|F(f)\|_{y} \leqq \varepsilon\|F\|$. Since $\varepsilon$ is arbitrary, we conclude that $F(f)=0$ on $X_{y}$.

Lemma 7.11. Let $M$ be a $G$-invariant $B$-submodule of $A$ of constant finite dimension, and let $U_{y}$ be the vector space of restrictions to $X_{y}$. If $f \in M$ and $t \in \bar{G}$, then the function $f_{t} \mid X_{y}$ defined on $X_{y}$ as $\left(f_{t} \mid X_{y}\right)(x)=f(t x)$ is in $U_{y}$.

Proof. Let $g_{n} \rightarrow t$. Then, for $x$ in $X_{y}, f_{g_{n}}(x) \rightarrow f_{t}(x)$ uniformly for $x$ in $X_{y}$ by Lemma 5.1. Since $f_{g_{n}} \mid X_{y}$ is in $U_{y}$ and since $U_{y}$ is finite-dimensional $f_{t} \mid X_{y}$ is in $U_{y}$. 
Proposition 7.12. Let $M$ and $N$ be G-invariant B-submodules of $A$ of constant finite dimension, and let $F$ be an equivalence of $M$ onto $N$. If $U_{y}$ and $V_{y}$ are the respective vector spaces of restrictions to $X_{y}$, then the induced map $F^{y}$ exhibits $U_{y}$ and $V_{y}$ as equivalent under the action of $I_{y}$.

Proof. If we apply Lemma 7.10 to the bounded operator $F^{-1}$, we see that $\left(F^{-1}\right)^{y}$ exists. It is clear that $\left(F^{-1}\right)^{y}$ is a two-sided inverse of $F^{y}$. Lemma 7.11 shows that $U_{y}$ and $V_{y}$ are invariant under $I_{y}$.

Now let $f$ be in $U_{y}$, let $s$ be in $\bar{I}_{y}$, and let $g_{n} \rightarrow s$. We are to show that

$$
F^{y}\left(f_{s}\right)=F^{y}(f)_{s} \text {. }
$$

Extend $f$ to a member $h$ of $M$. Since $F^{y}$ is bounded and since $h_{g_{n}}(x) \rightarrow f_{s}(x)$ uniformly for $x$ in $X_{y}$, we have

$$
F^{y}\left(f_{s}\right)(x)=\lim F^{y}\left(h_{g_{n}} \mid X_{y}\right)(x) .
$$

On the other hand,

$$
F^{y}\left(h_{g_{n}} \mid X_{y}\right)(x)=F\left(h_{g_{n}}\right)(x)=F(h)_{g_{n}}(x)=F(h)\left(g_{n} x\right)
$$

and

$$
\lim F(h)\left(g_{n} x\right)=F(h)(s x)=F^{y}(f)_{s}(x)
$$

Hence

$$
F^{y}\left(f_{s}\right)=F^{y}(f)_{s} .
$$

Proposition 7.13. Let $M$ and $N$ be G-invariant B-submodules of $A$ of constant finite dimension, and let $U_{e}$ and $V_{e}$ be the respective vector spaces of restrictions to $X_{e}$. If $H$ is a linear transformation of $U_{e}$ into $V_{e}$ which commutes with $I_{e}$, then there exists a bounded B-linear transformation of $M$ into $N$ which commutes with $G$ and satisfies $F^{e}=H$.

Proof. If $f$ is in $M$ and $t$ is in $\bar{G}$, then Lemma 7.11 shows that $f_{t} \mid X_{e}$ is in $U_{e}$. We therefore define

$$
F(f)(t e)=H\left(f_{t} \mid X_{e}\right)(e)
$$

First we show the definition is unambiguous. If $t e=t^{\prime} e$, then $t^{-1} t^{\prime}$ is in $\bar{I}_{e}$ and $\left(f_{t} \mid X_{e}\right)_{t^{-1} t^{\prime}}=f_{t^{\prime}} \mid X_{e}$. Hence the given commutativity of $H$ with $I_{e}$ implies that

$$
\begin{aligned}
F(f)\left(t^{\prime} e\right) & =H\left(f_{t^{\prime}} \mid X_{e}\right)(e)=H\left(\left(f_{t} \mid X_{e}\right)_{t^{-1} t^{\prime}}\right)(e) \\
& =H\left(f_{t} \mid X_{e}\right)\left(t^{-1} t^{\prime} e\right)=H\left(f_{t} \mid X_{e}\right)(e)=F(f)(t e),
\end{aligned}
$$

and the definition is consistent.

For continuity of $F(f)$, let $x_{n}=t_{n} e \rightarrow x$. If continuity fails, choose a subnet of $x_{n}$ such that $F(f)\left(x_{n}\right)$ converges to something other than $F(f)(x)$, and extract a further subnet so that $t_{n}$ converges to some $t$ in $\bar{G}$. The desired contradiction follows from Lemma 5.1 and the boundedness of $H$. 
It is clear that $F$ is $B$-linear and that $\|F\| \leqq\|H\|$. Since

$$
F(f)(g t e)=H\left(f_{g t} \mid X_{e}\right)(e)=H\left(\left(f_{g}\right)_{t} \mid X_{e}\right)(e)=F\left(f_{g}\right)(t e),
$$

$F$ commutes with $G$. Also $F^{e}=H$ because

$$
F^{e}\left(f \mid X_{e}\right)(s e)=F(f)(s e)=H\left(f_{s} \mid X_{e}\right)(e)=H\left(f \mid X_{e}\right)(s e) .
$$

Finally we are to show that $F$ has range in $N$. By remark (3) it suffices to show that, for $f \in M$ and $y \in M(B), F(f) \mid X_{y}$ lies in the space $V_{y}$ of restrictions of $N$ to $X_{y}$. First notice that if $\pi(t e)=y$ and $p=s e$ is in $X_{e}$, then

$$
F(f)(t p)=H\left(f_{t s} \mid X_{e}\right)(e)=H\left(\left(f_{t}\right)_{s} \mid X_{e}\right)(e)=H\left(f_{t} \mid X_{e}\right)(p) .
$$

This observation applied to $t^{-1} x$ for $x$ in $X_{y}$ shows that

$$
F(f)(x)=H\left(f_{t} \mid X_{e}\right)\left(t^{-1} x\right) .
$$

Let $h$ be an extension of $H\left(f_{t} \mid X_{e}\right)$ to a function in $N$, and let $g_{n} \rightarrow t^{-1}$ in $\bar{G}$. Then $h_{g_{n}} \mid X_{y}$ is in $V_{y}$ and hence so is the uniform limit, which is $F(f) \mid X_{y}$.

THEOREM 7.14. If $M$ is an irreducible $G$-invariant B-submodule of $A$ of constant finite dimension, then there is a $\lambda$ such that $M \subseteq$ image $\left(P_{\lambda}\right)$. If $M$ and $N$ are two such modules, then $M$ and $N$ are equivalent if and only if they are contained in the same image $\left(P_{\lambda}\right)$.

REMARK. This theorem is a corrected version of conclusion (2) of Theorem 3 in [9].

Proof. By Proposition 7.8, there is a $\lambda$ and there is an irreducible $G$-invariant $B$-submodule $R$ such that $M$ is equivalent to $R$ and $R \subseteq$ image $\left(P_{\lambda}\right)$. Apply Proposition 7.12. In the notation of that proposition, $U_{y}$ is equivalent to $V_{y}$ for each $y$. But $V_{y}$ is contained in the restriction $W_{y}$ of image $\left(P_{\lambda}\right)$ to $X_{y}$, and $W_{y}$ is such that it contains every vector space of functions on $X_{y}$ equivalent to any of its subspaces. Hence $U_{y} \subseteq W_{y}$ and $M \subseteq \operatorname{image}\left(P_{\lambda}\right)$.

If $N$ is given and $N$ is equivalent to $M$, then $N$ is equivalent to $R$ and the same argument shows that $N \subseteq$ image $\left(P_{\lambda}\right)$.

Let $M$ and $N$ be irreducible and contained in $W=\operatorname{image}\left(P_{\lambda}\right)$. If $U_{e}$ and $V_{e}$ are the respective spaces of restrictions to $X_{e}$, then $U_{e}$ and $V_{e}$ are invariant under $I_{e}$ by Lemma 7.11. Write $U_{e}$ and $V_{e}$ each as the direct sum of irreducible $I_{e}$-invariant vector spaces. Any two such spaces, being in $W_{e}$, are equivalent. Hence we can construct, summand by summand, a nonzero linear transformation $H$ of $U_{e}$ into $V_{e}$ which commutes with $I_{e}$. Apply Proposition 7.13 to get a nonzero bounded $B$-linear map $F$ of $M$ into $N$ which commutes with $G$. By Lemma 7.7, $M$ and $N$ are equivalent.

8. Crossed representations. Step three in the treatment of distal functions is to analyze the individual $G$-invariant $B$-modules of constant finite dimension. Again let $B$ be a distal algebra, but now let $A=A P(B)$. Let $\pi$ be the projection of $M(A P(B))$ onto $M(B)$. 
We shall approach this problem in two ways. Both ways begin by associating to each such module a vector bundle with base space $M(B)$. The first way, which is coordinate free, further defines on the bundle a "unitary" fiber-preserving action of $G$; we shall consider these $G$-vector bundles only briefly. The second way, which uses coordinates and is more appropriate for $\S 9$, uses matrices to express the action of $G$ and associates to each module a "unitary crossed representation." The functions of the module can be recovered from this representation, and, in fact, the functions arising from any abstract unitary crossed representation are all almost periodic over $B$ and together form a $G$-invariant $B$-module of constant finite dimension. When $B$ is the algebra of constants, the unitary crossed representations reduce to ordinary unitary representations of $G$, and our results reduce to familiar theorems about almost periodic functions.

The problem of classifying the crossed representations involves the problem of deciding when two crossed representations lead to the same module of functions, and this problem of equivalence is treated briefly at the end of the section.

We begin by associating to each $G$-invariant $B$-submodule $M$ of $A P(B)$ of constant dimension a complex vector bundle with base space $M(B)$ in such a way that the continuous cross-sections of the bundle are identified with the members of $M$.

Let $M$ be a $G$-invariant $B$-submodule of $A P(B)$ of constant dimension $n$; the module $M$ will be fixed until further notice. Let $\mathscr{M}$ denote the set of all restrictions of members of $M$ to individual fibers of $M(A P(B))$, and let the restrictions to $X_{y}$ $=\pi^{-1}(y)$ be denoted by $V_{y}$. We define a topology on $\mathscr{M}$ as follows: If $f$ is a restriction to the fiber $X_{y}$, a subbase open set containing $f$ is any set $N_{f}\left(f_{1}, U, \varepsilon\right)$ such that

(a) $\varepsilon$ is a positive real number, $U$ is an open neighborhood of $y$ in $M(B)$, and $f_{1}$ is a member of $M$ such that $f=f_{1} \mid X_{y}$.

(b) $N_{f}\left(f_{1}, U, \varepsilon\right)=\left\{h \in V_{z}\left|z \in U, \sup _{x \in X_{z}}\right| f_{1}(x)-h(x) \mid<\varepsilon\right\}$.

Lemma 7.9 can be used to show that this class of sets is actually a base for the topology, but this fact will not be needed.

If $f$ is a restriction to $X_{y}$, we define $p(f)=y$. Then $p$ is a continuous projection of $\mathscr{M}$ onto $M(B)$, because the inverse image of an open set $U$ in $M(B)$ is $\bigcup_{k=1}^{\infty} N(0, U, k)$. The inverse image of a single point of $M(B)$ is a complex vector space isomorphic to $C^{n}$.

Now we make $\mathscr{M}$ into a bundle. The bundle space is $\mathscr{M}$, the base space is $M(B)$, the projection is $p$, the fiber is $C^{n}$, and the structure group is the group $U(n)$ of $n$-by- $n$ unitary matrices. Let $\left\{f_{i}^{U} \mid 1 \leqq i \leqq n, U \in\right.$ finite cover $\}$ be a local orthonormal base for $M$. We define a coordinate function $\phi_{U}: U \times C^{n} \rightarrow p^{-1}(U)$ by

$$
\phi_{U}\left(y,\left[c_{1}, \ldots, c_{n}\right]\right)=\sum_{i=1}^{n} c_{i}\left(f_{i}^{U} \mid X_{y}\right) .
$$

It is clear that $\phi_{U}$ is fiber-preserving, and Proposition 8.1 will show that $\phi_{U}$ is a homeomorphism. Set $\phi_{U, y}(c)=\phi_{U}(y, c)$, and, if $y \in U \cap V$, define a matrix $h^{U V}(y)$ by

$$
f_{i}^{U} \mid X_{y}=\sum_{j} h_{i j}^{U V}(y)\left(f_{j}^{V} \mid X_{y}\right)
$$


Then

$$
\phi_{\bar{V}, y}^{-1} \phi_{U, y}\left[c_{1}, \ldots, c_{n}\right]=\left[\sum_{i} c_{i} h_{i 1}^{U V}, \ldots, \sum_{i} c_{i} h_{i n}^{U V}\right],
$$

and hence $h^{U V}(y)$, when thought of as acting on row vectors, equals the coordinate transformation $\phi_{V, y}^{-1} \phi_{U, y}$. The matrix $h^{U V}(y)$ is unitary, and Lemma 6.2 shows that the dependence on $y$ is continuous. Thus $\mathscr{M}$ becomes a coordinate bundle under this structure. Any two coordinate bundle structures induced by different local orthonormal bases are clearly equivalent, and hence $\mathscr{M}$ inherits the structure of a fiber bundle. Proposition 8.2 will identify the continuous cross-sections of $\mathscr{M}$ with the members of the module $M$.

Proposition 8.1. $\phi_{U}: U \times C^{n} \rightarrow p^{-1}(U)$ is a homeomorphism.

Proof. For continuity of $\phi_{U}$, let $y_{k} \rightarrow y_{0} \in U$ and $c_{j}^{k} \rightarrow c_{j}$ for $j=1, \ldots, n$. Let $N\left(f_{1}, V, \varepsilon\right)$ be a neighborhood of $\sum c_{j}\left(f_{j}^{U} \mid X_{y_{0}}\right)$. Then

$$
\left\|\sum c_{j} f_{j}^{U}-\sum c_{j}^{k}\left(f_{j}^{U} \mid X_{y_{k}}\right)\right\|_{y_{k}} \leqq \sum_{j}\left|c_{j}-c_{j}^{k}\right|\left\|f_{j}^{U}\right\|_{y_{k}},
$$

and the right side is eventually less than $\varepsilon / 2$ by Lemma 7.9. Also Lemma 7.9 shows that, for some neighborhood $W$ of $y_{0}$,

$$
\left\|f_{1}-\sum c_{j} f_{j}^{U}\right\|_{W}<\varepsilon / 2
$$

and hence

$$
\left\|f_{1}-\sum c_{j} f_{j}^{U}\right\|_{y_{k}}<\varepsilon / 2
$$

eventually. So eventually $\sum c_{j}^{k}\left(f_{j}^{U} \mid X_{y_{k}}\right)$ is in $N\left(f_{1}, V, \varepsilon\right)$.

For continuity of $\phi_{U}^{-1}$, we need show only that a net in the locally compact space $U \times C^{n}$ which diverges to infinity cannot be mapped by $\phi_{U}$ into a convergent net. (In fact, then $\phi_{U}$ extends to be continuous on the one-point compactification.) Composition with $p$ shows that the $U$ component of such a net in $U \times C^{n}$ must converge to something in $U$. Hence the $C^{n}$ component diverges to infinity. Thus suppose $\left[c_{1}^{k}, \ldots, c_{n}^{k}\right]$ diverges and $\sum c_{j}^{k}\left(f_{j}^{U} \mid X_{y_{k}}\right)$ converges. Since the square root of the sum of the squares of the $c$ 's is diverging to infinity, it suffices in an obvious notation to prove that $E\left(|\cdot|^{2} \mid B\right)^{1 / 2}$ is a continuous complex-valued function on $\mathscr{M}$.

Let $f$ be in $\mathscr{M}$ with $p(f)=y_{0}$, and let $\varepsilon>0$ be given. Let $f_{1}$ be a fixed extension of $f$, and let $V$ be an open neighborhood of $y_{0}$ such that, for $y \in V$,

$$
\left|E\left(\left|f_{1}\right|^{2} \mid B\right)^{1 / 2}\left(y_{0}\right)-E\left(\left|f_{1}\right|^{2} \mid B\right)^{1 / 2}(y)\right|<\varepsilon / 2 .
$$

We claim that $N_{f}\left(f_{1}, V, \varepsilon / 2\right)$ is mapped into the $\varepsilon$-neighborhood of $E\left(|f|^{2} \mid B\right)^{1 / 2}\left(y_{0}\right)$. In fact, if $h$ is in $N_{f}\left(f_{1}, V, \varepsilon / 2\right)$ with $p(f)=z$, then

$$
\begin{aligned}
\left|E\left(\left|f_{1}\right|^{2} \mid B\right)^{1 / 2}(z)-E\left(|h|^{2} \mid B\right)^{1 / 2}(z)\right| & \leqq E\left(\left|f_{1}-h\right|^{2} \mid B\right)^{1 / 2}(z) \\
& \leqq\left\|f_{1}-h\right\|_{z}<\varepsilon / 2
\end{aligned}
$$

and so (8.2), with $y=z$, gives

$$
\left|E\left(|f|^{2} \mid B\right)^{1 / 2}\left(y_{0}\right)-E\left(|h|^{2} \mid B\right)^{1 / 2}(z)\right|<\varepsilon .
$$


Proposition 8.2. If $q$ is a continuous function from $M(B)$ into $\mathscr{M}$ such that $p q$ is the identity on $M(B)$, then the function defined on $M(A P(B))$ by

$$
h(x)=q(\pi(x))(x)
$$

is a member of $M$. Every element of $M$ arises in this way from such a function $q$.

Proof. In view of remark (3) at the beginning of $\S 7, h$ will be in $M$ if $h$ is continuous on $M(A P(B))$. Thus let $x_{n} \rightarrow x$ and let $\varepsilon>0$ be given. Fix an extension $f_{1}$ of $q(\pi(x))$. Eventually $\left|f_{1}\left(x_{n}\right)-f_{1}(x)\right|$ is $<\varepsilon / 2$ and also, since $q \pi$ is continuous, $q\left(\pi\left(x_{n}\right)\right)$ is in the neighborhood $N\left(f_{1}, M(B), \varepsilon / 2\right)$ of $q(\pi(x))$. Then for large $n$

$$
\begin{aligned}
\left|q\left(\pi\left(x_{n}\right)\right)\left(x_{n}\right)-q(\pi(x))(x)\right| \leqq & \left\|q\left(\pi\left(x_{n}\right)\right)-\left(f_{1} \mid X_{\pi\left(x_{n}\right)}\right)\right\| \\
& +\left|f_{1}\left(x_{n}\right)-f_{1}(x)\right|<\varepsilon .
\end{aligned}
$$

Hence $h$ is continuous and is a member of $M$.

Conversely let $h$ be given and put $q(y)=h \mid X_{y}$. We are to show that $q$ is continuous. Let $y_{n} \rightarrow y$ and let $N_{q(y)}\left(f_{1}, U, \varepsilon\right)$ be a neighborhood of $q(y)$. Since $h$ and $f_{1}$ agree on $X_{y}$, Lemma 7.9 shows that there is an open subneighborhood $W$ of $U$ containing $y$ such that $\left\|h-f_{1}\right\|_{w}<\varepsilon$. If $n$ is chosen large enough so that $y_{n}$ is in $W$, then $q\left(y_{n}\right)$ will be in $N_{q(y)}\left(f_{1}, U, \varepsilon\right)$. The proof is complete.

The action of $G$ on $M$ has not yet appeared in the properties of $\mathscr{M}$. The coordinate-free way of bringing it in is to define an action of $G$ on the vector bundle and arrive at what might be called a G-vector bundle. If $f$ is defined on $X_{y}$ and $x$ is in $X_{g y}$, put

$$
g\left(f \mid X_{y}\right)(x)=f\left(g^{-1} x\right) .
$$

The result is defined on $X_{g y}$. It is easily seen that this definition gives a left action of $G$ on $\mathscr{M}$ with these two characteristic properties:

(a) The action commutes with $p$ and is jointly continuous.

(b) The action of $g$ restricted to $V_{y}$ is a linear transformation onto $V_{g y}$ preserving the inner product obtained from $E(\cdot \mid B)$.

We omit the details.

The approach with coordinates brings in the action of $G$ by means of a crossed representation. Fix a coordinate system for $\mathscr{M}$ corresponding to a local orthonormal base $\left\{f_{i}^{U}\right\}$. If $g$ is in $G$, if $y$ is in $U$, and if $g y$ is in $V$, let $D^{V U}(g, y)$ be the matrix defined by

$$
f_{i}^{V}(g x)=\sum_{j=1}^{n} D_{i j}^{V U}(g, y) f_{j}^{U}(x), \quad x \in X_{y}
$$

The assumption that the $\left\{f_{i}^{U}\right\}$ are orthonormal implies that the matrices $D^{V U}(g, y)$ are all unitary.

The reader is asked to keep in mind the case in which $\mathscr{M}$ is a product bundle; $\mathscr{M}$ is a product bundle if and only if the cover $\{U\}$ can be taken to consist of just the one set $M(B)$. In this case there is a global orthonormal base $\left\{f_{i}\right\}$, and the set 
of matrices $D(g, y)$ defined in (8.3) gives a crossed homomorphism (actually continuous) of $G$ into unitary-matrix-valued functions on $M(B)$. The classical situation with almost periodic functions is a special instance. Then $B$ is the algebra of constants and $M(B)$ consists of one point. Each bundle is a product bundle, and the matrices $D(g, y)$ reduce to a continuous group representation of $G$ in $U(n)$.

Proposition 8.3. The matrices $D^{V U}(g, y)$ are unitary and satisfy these five properties:

(a) If $y \in U \cap U^{\prime}$ and $g y \in V$,

$$
D^{V U^{\prime}}(g, y)=D^{v U}(g, y) h^{U U^{\prime}}(y) .
$$

(b) If $y \in U$ and $g y \in V \cap V^{\prime}$,

$$
D^{V^{\prime} U}(g, y)=h^{V^{\prime} V}(g y) D^{V U}(g, y) .
$$

(c) If $y \in U$, hy $\in V$, and $g h y \in W$,

$$
D^{W U}(g h, y)=D^{W v}(g, h y) D^{v U}(h, y) .
$$

(d) If $y \in U, D^{U U}(e, y)=I$.

(e) $D^{V U}(g, y)$ is jointly continuous in the variables $g$ and $y$.

Proof. It was noted above that the matrices are unitary. Properties (a) and (b) follow directly from (8.1) and (8.3), while (c) and (d) use (8.3) alone. We sketch a proof of (e). Let $g_{n} \rightarrow g$ and $y_{n} \rightarrow y$, and let (8.3) hold. Then

$$
D_{i j}^{V U}(g, y)=E\left(f_{i}^{V}(g, x) \bar{f}_{j}^{U}(x) \mid B\right)(y),
$$

and a similar relation holds for $D^{V U}\left(g_{n}, y_{n}\right)$. Since we are interested in what happens only in a neighborhood of $y$, we may replace the $f$ 's with globally defined continuous functions which agree with the $f$ 's on all fibers in a neighborhood of $y$. We give the new functions the same names. On one hand the functions $f_{i}^{V}$ are uniformly continuous, and on the other hand $E\left(\left(f_{i}^{V}\right)_{g} \bar{f}_{j}^{U} \mid B\right)$ is continuous on $M(B)$. Thus a $2 \varepsilon$-argument completes the proof.

A set of unitary matrices $D^{V U}(g, y)$ associated to a $U(n)$ vector bundle over $M(B)$, defined whenever $y \in U$ and $g y \in V$, and satisfying properties (a) through (e) of Proposition 8.3 is called a unitary crossed representation of $G$ relative to $B$.

In the crossed representation constructed above, the matrices $D^{V U}(g, y)$ and the $n$ numbers $f_{i} U_{0}(e), 1 \leqq i \leqq n$, for any fixed $U_{0}$ containing $e$ determine the module $M$ completely. To prove this statement, we proceed as follows. Let $V$ be given and let $x \in \pi^{-1}(V)$. Choose a net $\left\{g_{m}\right\}$ with $g_{m} \rightarrow x$. For large $m, D^{V U_{0}}\left(g_{m}, e\right)$ is defined and we have

$$
f_{i}^{V}(x)=\lim _{m} \sum_{j} D_{i j}^{V U_{0}}\left(g_{m}, e\right) f_{i}^{U_{0}}(e) .
$$

It is reasonable that other choices of the constants $f_{j}^{U}(e)$ will lead to $G$-invariant $B$-submodules of $A P(B)$. We are now going to prove that every choice of these 
constants leads to such a submodule. The span of these modules will be called the set of functions determined by $\left\{D^{v U}(g, y)\right\}$. The result we shall therefore establish is the following:

PROPOSITION 8.4. If $\left\{D^{V U}(g, y)\right\}$ is any unitary crossed representation of $G$ relative to $B$, then the set of functions determined by $\left\{D^{V U}(g, y)\right\}$ is a G-invariant B-submodule of $A P(B)$ of constant finite dimension.

The proof will be given after some preliminary discussion. The main difficulty will be to show that the functions in the module are distal, and the proof will be roundabout. We begin by constructing a flow from the crossed representation.

Let $\mathscr{M}$ be the underlying vector bundle of $\left\{D^{v U}(g, y)\right\}$. We form a fiber bundle which is the transpose inverse of the principal bundle of $\mathscr{M}$. If $J$ is the index set for the cover $\{U\}$, the bundle space is all equivalence classes in $M(B) \times U(n) \times J$ where $\left(y_{1}, u_{1}, U\right) \sim\left(y_{2}, u_{2}, V\right)$ if $y_{1}=y_{2}$ and $u_{2}=h^{v U}\left(y_{1}\right) u_{1}$, the base space is $M(B)$, the fiber is $U(n)$, and the projection $\bar{p}$ satisfies $\bar{p}\left(y_{1}, u_{1}, U\right)=y_{1}$. This fiber bundle we shall call $P(\mathscr{M})$. Its topology is compact Hausdorff. We let $\left\{\psi_{U}\right\}$ be its set of coordinate functions.

LEMMA 8.5. The identity

$$
g \psi_{U}(y, k)=\psi_{V}\left(g y, D^{v U}(g, y) k\right), \quad \text { where } y \in U, g y \in V, k \in U(n),
$$

unambiguously defines a jointly continuous group action of $G$ in $P(\mathscr{M})$.

Proof. Let

$$
\psi_{U}(y, k)=\psi_{U}\left(y, k^{\prime}\right)
$$

and let $g y \in V^{\prime}$. To prove that the definition is unambiguous, we must show that

$$
\psi_{V}\left(g y, D^{v U}(g, y) k\right)=\psi_{V^{\prime}}\left(g y, D^{V^{\prime} U^{\prime}}(g, y) k^{\prime}\right) .
$$

From (8.4) we have $k=h^{U U^{\prime}}(y) k^{\prime}$, and this equation, together with (a) and (b) of Proposition 8.3, gives

$$
D^{V U}(g, y) k=h^{V V^{\prime}}(g y) D^{V^{\prime} U^{\prime}}(g, y) k^{\prime},
$$

which is equivalent with (8.5). The given transformations form a group action by (c) and (d) of Proposition 8.3, and the action is jointly continuous by (e) of that proposition. The proof is complete.

Since $P(\mathscr{M})$ is compact Hausdorff, $(P(\mathscr{M}), G)$ is a flow. Let $d$ be a left-invariant metric for $U(n)$, and let $\Delta$ denote the set of pairs $\left(x_{1}, x_{2}\right)$ such that $x_{1}$ and $x_{2}$ are in the same fiber of $P(\mathscr{M})$. Define a function $\rho$ from $\Delta$ to the reals by

$$
\rho\left(\psi_{U}(y, k), \psi_{U}\left(y, k^{\prime}\right)\right)=d\left(k, k^{\prime}\right) .
$$

To see that this definition is unambiguous, let $\psi_{U}(y, k)=\psi_{V}\left(y, k_{1}\right)$ and $\psi_{U}\left(y, k^{\prime}\right)$ $=\psi_{V}\left(y, k_{1}^{\prime}\right)$. Then

$$
k_{1}=h^{V U}(y) k \quad \text { and } \quad k_{1}^{\prime}=h^{V U}(y) k^{\prime} .
$$


Since $d$ is left-invariant, $d\left(k_{1}, k_{1}^{\prime}\right)=d\left(k, k^{\prime}\right)$. Thus $\rho$ is unambiguous. It is clear that $\rho$ defines a metric on each fiber and that $\rho$ is continuous on $\Delta$. Moreover $\rho$ is $G$-invariant. In fact, let $y \in U$ and $g y \in V$. Then

and

$$
\rho\left(\psi_{U}(y, k), \psi_{U}\left(y, k^{\prime}\right)\right)=d\left(k, k^{\prime}\right)
$$

$$
\rho\left(g \psi_{U}(y, k), g \psi_{U}\left(y, k^{\prime}\right)\right)=d\left(D^{v U}(g, y) k, D^{v U}(g, y) k^{\prime}\right) .
$$

These two expressions are equal because $d$ is left-invariant.

We need the following result proved by Furstenberg in [7].

Lemma 8.6. An isometric extension of a distal flow is distal.

Proof. Let $(X, G)$ and $(Y, G)=\pi(X, G)$ be flows with $Y$ distal, and let $\rho^{*}$ exhibit the isometric extension. Let $g_{n} x \rightarrow z$ and $g_{n} x^{\prime} \rightarrow z$. Then $g_{n} \pi(x) \rightarrow \pi(z)$ and $g_{n} \pi\left(x^{\prime}\right)=\pi(z)$. Since $Y$ is distal, $\pi(x)=\pi\left(x^{\prime}\right)$. Hence $\rho^{*}\left(x, x^{\prime}\right)$ is defined. But then

$$
0=\rho^{*}(z, z)=\lim \rho^{*}\left(g_{n} x, g_{n} x^{\prime}\right)=\rho^{*}\left(x, x^{\prime}\right)
$$

by the continuity and $G$-invariance of $\rho^{*}$. Hence $x=x^{\prime}$.

Lemma 8.7. Let $x_{0}$ be any point in $\bar{p}^{-1}(e)$. If $x_{0}$ is taken as the evaluation-atidentity element of $P(\mathscr{M})$, then every continuous complex-valued function on $P(\mathscr{M})$ restricts to a function on $G$ which is almost periodic relative to $B$.

Proof. The function $\rho$ exhibits $P(\mathscr{M})$ as an isometric extension of $M(B)$. By Proposition 3.5 and Lemma 8.6, the functions arising from the orbit of $x_{0}$ in $P(\mathscr{M})$ are all distal. Since $x_{0}$ lies in the fiber above $e$ in $M(B)$, Lemma 4.8 applies and shows that the functions are all a.p. over $B$.

Proof of Proposition 8.4. Let $U_{0}$ be a fixed open set in the cover which contains $e$ in $M(B)$. Fix an open set $V$ in the cover of $M(B)$ and let $y$ be a point of $V$. Let $f: M(B) \rightarrow[0,1]$ be a continuous function whose closed support is in $V$ and which is one in a neighborhood of $y$. (This neighborhood is to be thought of as nearly all of $V$.) The globally defined function

$$
f(g) D_{i j}^{V U_{0}}(g, e)
$$

is the restriction to the orbit of $\psi_{U}(e, I)$ of a continuous function on $P(\mathscr{M})$ and is therefore a.p. over $B$ by Lemma 8.7. Thus if $x \in X_{y}$ and if $g_{m}$ is a net with limit $x$, the limit

$$
\lim _{m} D_{i j}^{V U_{0}}\left(g_{m}, e\right)
$$

exists since $f(\pi(x))=f(y)=1$. Therefore as $x, y$, and $f$ vary, we see that $D_{i j}^{V U_{0}}(g, e)$ is uniformly continuous for $g$ in any compact subset of $\pi^{-1}(V)$. Consequently the functions determined by $\left\{D^{V U}(g, y)\right\}$ are all a.p. over $B$. The space of restrictions of such functions to any fiber is of dimension at most $n^{2}$, and hence to complete the proof it is sufficient to show that this set of functions is $G$-invariant. That is, 
it suffices to show that for fixed $h$ the function $D_{i j}^{W U}(h g, e)$ is uniformly continuous for $h g$ in any compact subset of $\pi^{-1}(W)$. Thus let $g_{m} \rightarrow x$ and let $\pi(x) \in V$. For large $m$

and hence

$$
D^{W U_{0}}\left(h g_{m}, e\right)=D^{W V}\left(h, g_{m}\right) D^{V U_{0}}\left(g_{m}, e\right)
$$

$$
\lim _{m} D^{W U_{0}}\left(h g_{m}, e\right)=D^{W v}(h, \pi(x)) \lim D^{v U_{0}}\left(g_{m}, e\right)
$$

Since for fixed $h, D^{w V}(h, \pi(x))$ is continuous for $\pi(x) \in V \subseteq M(B)$, the uniform continuity follows, and the proof is complete.

We conclude by characterizing equivalence of $B$-modules, as defined in $\S 7$, in terms of their induced crossed representations.

Proposition 8.8. Let $M$ and $\bar{M}$ be two G-invariant B-submodules of $A P(B)$ of constant dimension $n$, let $\left\{f_{i}^{U}\right\}$ and $\left\{\bar{f}_{i}^{\bar{U}}\right\}$ be local orthonormal bases for them, and let $\left\{D^{V U}(g, y)\right\}$ and $\left\{\bar{D}^{\nabla \bar{U}}(g, y)\right\}$ be the induced unitary crossed representations. Then $M$ and $\bar{M}$ are equivalent if and only if there exists a set of matrices $A^{U \bar{U}}(y)$ in $\operatorname{GL}(n, C)$ such that these four conditions are satisfied:

(a) $A^{U \bar{U}}(y)$ is defined whenever $U$ is a set in the open cover for $M, \bar{U}$ is a set in the open cover for $\bar{M}$, and $y \in U \cap \bar{U}$.

(b) $A^{U \bar{U}}(y)$ is continuous in $y$.

(c) The identities

$$
A^{U \bar{U}}(y)=h^{U U^{\prime}}(y) A^{U^{\prime}}(y)
$$

and

$$
A^{U \bar{U}^{\prime}}(y) h^{\bar{U}^{\prime} \bar{U}}(y)=A^{U \bar{U}}(y)
$$

hold whenever each side is defined.

(d) Whenever both sides are defined,

$$
\bar{D}^{\bar{V}}(g, y)=A^{V \bar{V}}(g y)^{-1} D^{V U}(g, y) A^{U \bar{U}}(y) .
$$

If $M$ and $\bar{M}$ are irreducible, then the matrices $A^{U \bar{U}}(y)$ must be a scalar times unitary matrices, where the scalar is independent of $y, U$, and $\bar{U}$. In any case if $M$ and $\bar{M}$ are equivalent, their associated vector bundles are equivalent.

Proof. If $F: M \rightarrow \bar{M}$ is an equivalence, then Lemma 7.10 shows that $F$ can be defined on the functions $f_{i}^{U}$. If $y \in U \cap \bar{U}$, define $A^{U \bar{U}}(y)$ by the identity

$$
F\left(f_{i}^{U}\right) \mid X_{y}=\sum_{j=1}^{n} A_{i j}^{U \bar{U}}(y)\left(\bar{f}_{j}^{\bar{D}} \mid X_{y}\right)
$$

Then (a) and (c) are immediate, (d) follows from the fact that $F$ commutes with $G$, and (b) follows from Lemma 6.2. Conditions (a), (b), and (c) define a vector bundle equivalence.

Conversely if the four conditions are satisfied, then (a) and the second half of (c) imply that the definition above of $F\left(f_{i}^{U}\right)$ makes sense. Condition (b) and Lemma 
7.9 imply that $F$ maps continuous functions into continuous functions and that $\|F\|_{y}$ is continuous. Since $M(B)$ is compact, $\|F\|$ is finite. Condition (d) implies that $F$ commutes with the action of the group. Reversing the argument and using the first half of (c) and the nonsingularity of $A^{U D}(y)$, we find that $F^{-1}$ is defined and bounded. Hence $F$ is one-one onto.

If matrices $A^{U \bar{U}}(y)$ exhibit irreducible $M$ and $\bar{M}$ as equivalent, then multiply both sides of (d) on the left by $A^{V \nabla}(g y)$ and on the right by $A^{U \bar{U}}(y)^{*}$. Use (c) in Proposition 8.3 and the unitary character of the $D$ 's and $\bar{D}$ 's to get

$$
D^{V U}(g, y)\left[A^{U \bar{U}}(y) A^{U \bar{U}}(y)^{*}\right]=\left[A^{V \bar{V}}(g y) A^{V \nabla}(g y)^{*}\right] D^{V U}(g, y) .
$$

The second half of (c) shows that the definition

$$
B^{U}(y)=A^{U \bar{U}}(y) A^{U \bar{U}}(y)^{*}
$$

is independent of $\bar{U}$, and the first half of (c) shows that

$$
B^{U}(y)=h^{U U^{\prime}}(y) B^{U^{\prime}}(y) h^{U U^{\prime}}(y)^{*} .
$$

If, for $U$ and $U^{\prime}$ in the cover for $M$, we define

$$
B^{U U^{\prime}}(y)=B^{U}(y) h^{U U^{\prime}}(y),
$$

then the $B$ 's clearly satisfy the second half of (c), and (8.8) can be used to show they satisfy the first half. On the other hand, (8.7) and the first two parts of Proposition 8.3 show that the $B$ 's satisfy (d) with $\bar{D}$ replaced by $D$. Thus the $B$ 's define an equivalence $F$ of the irreducible module $M$ with itself. By Lemma 7.7, $F=c I$ for some complex number $c$. Equation (8.6) applied to $B^{U U}=B^{U}$ shows that

$$
A^{U \bar{U}}(y) A^{U \bar{U}}(y)^{*}=c I .
$$

Since the $A$ 's are nonsingular, $c$ is real and $>0$. But then $A^{U D}(y)$ is $\sqrt{c}$ times a unitary matrix.

\section{Examples for $\$ 8$.}

EXAMPLE 1 (NILFLOWS AND A TWISTED BUNDLE). This example, which was constructed by $\mathrm{H}$. Furstenberg and L. Green, is a one-dimensional $B$-module whose underlying bundle is not a product bundle. The algebra $B$ will be a subalgebra of the almost periodic functions on the continuous reals.

Let $N$ be the nilpotent Lie group of matrices

$$
\left[\begin{array}{lll}
1 & x & y \\
0 & 1 & z \\
0 & 0 & 1
\end{array}\right],
$$

and let $\Gamma$ be the subgroup with $x, y$, and $z$ integers. If $N^{\prime}$ is the commutator subgroup of $N$, then it is easily checked that the group $N /\left(N^{\prime} \Gamma\right)$ is the 2-torus and the left coset space $\left(N^{\prime} \Gamma\right) / \Gamma$ is the circle. Hence the natural map of the left coset space $N / \Gamma$ onto $N /\left(N^{\prime} \Gamma\right)$ exhibits $N / \Gamma$ as a fiber bundle over the 2-torus with fiber 
$\left(N^{\prime} \Gamma\right) / \Gamma$, the circle. Thus $N / \Gamma$ is compact. Since the fundamental group of $N / \Gamma$ is the nonabelian group $\Gamma, N / \Gamma$ is not the 3-torus and the bundle is not a product bundle.

The continuous functions on $N / \Gamma$ arise from continuous functions on $N$ which are invariant under the transformations

$$
x \rightarrow x+a, \quad y \rightarrow y+b+c x, \quad z \rightarrow z+c,
$$

where $a, b$, and $c$ are integers. The function

$$
\exp (2 \pi i(y-x[z])) \sin 2 \pi z,
$$

where brackets denote integer part, is an example.

Direct calculation shows that the left action of $N$ on $N / \Gamma$ is distal and that $N / \Gamma$ is an isometric extension of $N /\left(N^{\prime} \Gamma\right)$. Hence, a fortiori, the same will be true of the action obtained by using only a subgroup of $N$. The subgroup we choose is the one-parameter subgroup $R$ of matrices

$$
\left[\begin{array}{ccc}
1 & t \alpha & \frac{1}{2} t^{2} \alpha \beta \\
0 & 1 & t \beta \\
0 & 0 & 1
\end{array}\right],
$$

where $\alpha$ and $\beta$ are fixed real numbers independent over the rationals. The $R$-orbits in $N / \Gamma$ are dense, and the left translates by members of $R$ (especially those with $t \beta$ slightly less than one) of the function in (9.1) separate points on $N / \Gamma$. It follows that the restriction to the $R$-orbit of $\Gamma$ of the function in (9.1), namely

$$
\exp \left(2 \pi i t \alpha\left(\frac{1}{2} t \beta-[t \beta]\right)\right) \sin 2 \pi t \beta,
$$

generates a distal algebra whose maximal ideal space is all of $N / \Gamma$. This algebra is almost periodic over the algebra $B$ of functions on the reals obtained from $N /\left(N^{\prime} \Gamma\right)$, namely those generated by continuous functions of period $\alpha^{-1}$ or $\beta^{-1}$. The $B$-module $M$ generated by the $R$-translates of the function in (9.2) gives rise to a flow in Lemma 8.5 which is a subflow of $N / \Gamma$. But since $M$ requires all of $N / \Gamma$ for the maximal ideal space of the algebra it generates, the flow in Lemma 8.5 is exactly $(N / \Gamma, R)$. Since $N / \Gamma$ is a twisted fiber bundle, the vector bundle associated to $M$ is twisted.

EXAMPLE 2 (REPRESENTATIONS WITH PRODUCT BUNDLES). Let us consider the general form of unitary crossed representations whose underlying bundle is a product bundle when the group $G$ is the integers. In this case a unitary crossed representation is a function $D(g, y)$ from $G \times M(B)$ into $n$-by- $n$ unitary matrices which is continuous in $y$ and which satisfies

$$
D(0, y)=I, \quad D(g+h, y)=D(g, h y) D(h, y) .
$$

The coordinate functions of the matrix $D(g, 0)$ generate as a $B$-module the functions arising from the representation, and this module is automatically invariant. By Proposition 8.4 these functions are a.p. over $B$. 
The whole representation is determined by $D(1, y)$, and we have, for $n>0$,

$$
\begin{aligned}
D(n, y) & =D(1,(n-1) y) \cdots D(1,1 y) D(1, y), \\
D(-n, y) & =D(1,(-n) y)^{-1} \cdots D(1,(-1) y)^{-1} .
\end{aligned}
$$

Conversely if $D(1, y)$ is given, these equations define a $D(g, y)$. So a representation is determined by a single continuous function from $M(B)$ into $U(n)$. Two are equivalent if there is a continuous $A(y)$ from $M(B)$ into $G L(n, C)$ such that

$$
E(g, y)=A(g y)^{-1} D(g, y) A(y) .
$$

That is, equivalence means

$$
E(1, y)=A(1 y)^{-1} D(1, y) A(y) .
$$

If $D$ and $E$ are irreducible, $A(y)$ may be assumed to be unitary.

If $D$ is one-dimensional, it is irreducible and $A$ may be taken to be unitary, i.e., of absolute value one. The one-dimensional representations form an abelian group under multiplication, a group of cocycles, and the trivial ones, those equivalent with the identity representation form a group of coboundaries. The quotient is a cohomology group equal to the group of equivalence classes of such representations. We have determined the group of cocycles above. For the coboundaries, the condition that $D(1, y)$ be trivial is that

$$
D(1, y)=A(1 y) / A(y)
$$

or that $D$ satisfy the functional equation

$$
D(1, n)=A(n+1) / A(n)
$$

for a function $A$ in $B$ of absolute value one.

Let $B$ be the algebra of all almost periodic functions. It is well known that if $a(n)$ is real-valued almost periodic and if its indefinite sum $b(n)$, with say $b(n+1)$ $-b(n)=a(n)$ and $b(0)=0$, is bounded, then $b(n)$ is almost periodic. Hence so is $e^{i b(n)}$. But what if $b(n)$ is unbounded? We assert that $e^{i b(n)}$ is at least a.p. over the almost periodic functions. In fact, if in (9.3) $y=0$ and $D(1, n)=e^{i a(n)}$, then $D(n, 0)=e^{i b(n)}$. The assertion follows.

For a final example, let $B$ again be the algebra of all almost periodic functions (or even just those generated by $e^{i n \theta}$ for some fixed $\theta$ ). If in $(9.3) y=0$ and $D(1, n)$ $=e^{i n \theta}$, then

$$
D(n, 0)=\exp \left(\frac{1}{2} \operatorname{in}(n-1) \theta\right)
$$

Therefore $\exp \left(\operatorname{in}^{2} \theta\right)$ is distal, as was asserted in $\S 3$. This method may be iterated to show that $e^{i p(n)}$ is distal for any real polynomial $p$.

\section{REFERENCES}

1. L. Auslander and F. Hahn, Real functions coming from flows on compact spaces and concepts of almost periodicity, Trans. Amer. Math. Soc. 106 (1963), 415-426. 
2. S. Bochner, A new approach to almost periodicity, Proc. Nat. Acad. Sci. U.S.A. 48 (1962), 2039-2043.

3. N. Dunford and J. T. Schwartz, Linear operators. Part I, Interscience, New York, 1958.

4. R. Ellis, Distal transformation groups, Pacific J. Math. 8 (1958), 401-405.

5. —, A semigroup associated with a transformation group, Trans. Amer. Math. Soc. 94 (1960), 272-281.

6. R. Ellis and W. H. Gottschalk, Homomorphisms of transformation groups, Trans. Amer. Math. Soc. 94 (1960), 258-271.

7. H. Furstenberg, Strict ergodicity and transformation of the torus, Amer. J. Math. 83 (1961), 573-601.

8. - The structure of distal flows, Amer. J. Math. 85 (1963), 477-515.

9. A. W. Knapp, Distal functions, Proc. Nat. Acad. Sci. U.S.A. 52 (1964), 1409-1412.

10. - Distal functions on abelian groups, Thesis, Princeton Univ., Princeton, N. J., 1965.

11. - Decomposition theorem for bounded uniformly continuous functions on a group, Amer. J. Math. 88 (1966), 902-914.

12. J. von Neumann, Almost periodic functions in a group. I, Trans. Amer. Math. Soc. 36 (1934), 445-492.

MassachusetTS INSTITUTE OF TeChNOLOGY,

CAMbridge, MasSaChusetTS 